\title{
Moduli and electromagnetic black brane holography
}

\author{
Dumitru Astefanesei, ${ }^{a, b}$ Nabamita Banerjee ${ }^{c}$ and Suvankar Dutta ${ }^{d}$ \\ ${ }^{a}$ Max-Planck-Institut für Gravitationsphysik, Albert-Einstein-Institut, \\ 14476 Golm, Germany \\ ${ }^{b}$ Erwin Scrödinger Inst., \\ 1090 Wien, Austria \\ ${ }^{c}$ Institute of Theoretical Physics, Utrecht University, \\ Leuvenlaan 4, 3584 CE Utrecht, The Netherlands \\ ${ }^{d}$ Department of Physics, Swansea University, \\ Singleton Park, Swansea, SA2 8PP, U.K. \\ E-mail: dumitru@aei.mpg.de, N.Banerjee@uu.nl, pysd@swan.ac.uk
}

\begin{abstract}
We investigate the thermodynamic and hydrodynamic properties of 4dimensional gauge theories with finite electric charge density in the presence of a constant magnetic field. Their gravity duals are planar magnetically and electrically charged AdS black holes in theories that contain a gauge Chern-Simons term. We present a careful analysis of the near horizon geometry of these black branes at finite and zero temperature for the case of a scalar field non-minimally coupled to the electromagnetic field. With the knowledge of the near horizon data, we obtain analytic expressions for the shear viscosity coefficient and entropy density, and also study the effect of a generic set of four derivative interactions on their ratio. We also comment on the attractor flows of the extremal solutions.
\end{abstract}

Keywords: AdS-CFT Correspondence, Brane Dynamics in Gauge Theories, Black Holes in String Theory

ARXIV EPRINT: 1008.3852 


\section{Contents}

1 Introduction 1

2 General set-up $\quad 3$

2.1 The model 4

2.2 Holographic data 6

3 Near horizon geometry $\quad 8$

3.1 Non-extremal case 9

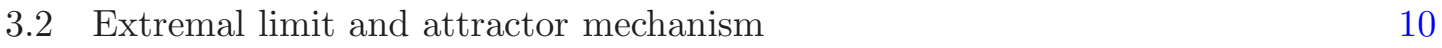

$\begin{array}{lll}3.2 .1 & \text { Attractor mechanism } & 11\end{array}$

$\begin{array}{ll}3.2 .2 \text { Entropy function } & 13\end{array}$

4 Shear viscosity to entropy density ratio $\quad 17$

4.1 Membrane paradigm and viscosity bound 18

$\begin{array}{lll}4.2 & \text { Four derivative action } & 20\end{array}$

5 Discussion $\quad 22$

$\begin{array}{ll}\text { A Kubo formula and shear viscosity } & 26\end{array}$

B Coefficients in expansion $(3.3)$ at $\mathcal{O}\left(r-r_{h}\right)^{2} \quad 29$

C Details on entropy function when the modulus is turned on 29

$\begin{array}{ll}\text { D Decoupling of } h_{x y} \text { mode } & 31\end{array}$

\section{Introduction}

The exploration of the phase diagram of Quantum Chromodynamics (QCD) (see, e.g., [1]) is a very active area of ongoing research in both, experimental and theoretical physics.

It is well known that the zero temperature ground state of QCD (at normal nuclear densities) has two important features: chiral symmetry is broken spontaneously and the color charges are confined to the interior of the individual hadrons (the scale is about 1 fermi). However, the studies in lattice QCD predicted that, beyond a temperature of about $150 \mathrm{MeV}$ (in fact, the critical temperature is $T_{c} \sim 200 \mathrm{MeV}$ ), the hadronic matter undergoes a transition from the confined phase to a deconfined color phase (of quarks and gluons). ${ }^{1}$

\footnotetext{
${ }^{1}$ Also, by increasing the temperature and/or density the QCD vacuum restores its chiral invariance.
} 
Since the transition temperature is not extremely high, it was expected that this phase of matter can even be produced in the laboratory. Indeed, the experimental data obtained at the Relativistic Heavy Ion Collider (RHIC) have confirmed the 'theoretical' expectation.

What came as a big surprise, though, is that the experimental data indicate that the quark-gluon plasma is a new state of thermalized matter, which exhibits almost ideal hydrodynamic behaviour. The early thermalization and a very small viscosity to entropy density area $(\eta / s)$ are characteristics of a strongly interacting system - at weak coupling $(g \ll 1)$, the equilibration time and ratio $\eta / s$ are proportional with the mean free path $\left(\sim 1 / g^{4}\right)$ and so they are parametrically large.

In other words, the experimental data are consistent with the interpretation of the new state as a strongly interacting plasma (see, e.g., [2] and references therein). This new phase of nuclear matter (the state of deconfinement super-hot hadronic matter) is known as the strongly coupled quark-gluon plasma (sQGP).

Obviously, the perturbation theory is not useful to investigate the properties of sQGP. Also, the lattice QCD study is not suitable for computing the dynamical quantities of sQGP. Interestingly enough, valuable guidance for understanding the near perfect fluidity observed at RHIC was obtained from the Anti-deSitter(AdS)/conformal field theory (CFT) duality [3]. ${ }^{2}$

It is important to emphasize that the gravity dual of QCD is not known. However, the QCD matter of interest is in a deconfined phase for which the 'conformal anomaly' (due to the running of the coupling) appears to be relatively small. Therefore, since QCD is approximately conformal at sufficiently large energies, one expects some similarities with some of the gauge theories that can be studied by using the AdS/CFT duality.

Indeed, a study of quantum field theories (QFT) with a gravity dual revealed the fact that, when heated up to finite temperature, these QFTs behave hydrodynamically (see, e.g., [5] and references therein and, also, [6]) at large distances and time scales.

The holographic hydrodynamics (the first attempt to study hydrodynamics via AdS/CFT was [7]) is an important tool for understanding some properties of strongly coupled quantum field theories in terms of AdS black holes physics. A notorious example is the computation of the ratio of the shear viscosity $(\eta)$ to the entropy density $(s)$. It was proposed in [8] that there is an universal viscosity bound, ${ }^{3}$ which is also satisfied by sQGP:

$$
\frac{\eta}{s} \geq \frac{\hbar}{4 \pi k_{B}} .
$$

Kubo formula relates the shear viscosity to the two-point function of energy momentum tensor in zero frequency limit (see appendix A for a review). From the field/operator correspondence of AdS/CFT duality, we know that the energy momentum tensor of boundary field theory is sourced by bulk graviton excitations. In [7], the authors have considered graviton excitations polarized parallel to the black brane, which are moving transverse to it. They found that the graviton absorption cross section is related to the shear viscosity coefficient.

\footnotetext{
${ }^{2} \mathrm{~A}$ concise review of phenomenological problems that can not be solved by the standard field theoretic approach, but for which the AdS/CFT duality may be useful, can be found in [4].

${ }^{3}$ However, there are known examples for which the bound is violated (see [9] and references therein).
} 
In this paper, we use holographic techniques to investigate the properties of 4dimensional gauge theories with finite electric charge density in the presence of a constant magnetic field. Strong magnetic fields are created in heavy ion collisions at RHIC, and some models were studied in [10-14].

A study of black branes solutions of Einstein-Maxwell AdS gravity with a gauge ChernSimons term can be found in [15-17]. We use the entropy function to carefully study the near horizon geometry of these black branes at zero temperature and discuss different branches with finite area horizons. This allows us to obtain an analytic expression for the entropy density that supports the numerical analysis of [17] (though, we find a larger class of near horizon geometries). That is, there is a critical value of the magnetic field for which the entropy vanishes.

We also extend the work of $[15,16]$ by including a modulus, which is exponentially coupled to the gauge field kinetic term in the action. This kind of coupling appears in consistent truncations of type $I I B$ supergravity on Sasaki-Einstein manifolds [20-23]. In the extremal limit, we argue that, due to the attractor mechanism, the near horizon geometry is stable under scalar fields fluctuations.

More importantly, unlike $[15,16]$, our main focus is on the hydrodynamics properties of this system. We use the membrane paradigm approach [24] and its higher derivative generalization $[25,26]$ (see, also, [27]) to compute the shear viscosity (related work for finite chemical potential can be found in [28-37]). In particular, we study the effect of the moduli (in the presence of a generic set of four-derivative interactions) on the viscosity bound.

Our results are given in terms of near horizon data, and not in terms of the field theory parameters that would be measured at the $A d S_{5}$ boundary. For a computation of the viscosity of our system, that is enough. However, there is a non-trivial flow for other transport coefficients, e.g. the conductivity. Also, it will be interesting to express the entropy in terms of asymptotic parameters - in [17] this problem was solved analytically (for vanishing scalar field). We will present some of these results in the forthcoming work [18, 19].

An overview of the paper is as follows: In section 2, we present the bulk action, set-up the conventions for our model, and provide some useful holographic data. In section 3 , we carefully study the near horizon geometry of electromagnetic black branes at zero and finite temperature. In the extremal limit, we use the entropy function and present exact near horizon geometry solutions. In section 4 , we compute the shear viscosity to entropy density in the presence of a generic set of four derivative interactions. Finally, we conclude with a discussion of our results and present some future directions. The appendices contain supporting material.

\section{General set-up}

The AdS/CFT duality provides a concrete relation between the regular black brane solutions in $A d S_{5}$ and the hydrodynamic regime of strongly coupled 4-dimensional quantum field theories.

In this section, we fix the conventions for our model and present a brief review of holography relevant to our work. We follow closely $[15,16]$, though our model is different 
because we also consider scalar fields. In this way, it is straightforwardly to compare some of the results. However, one important difference is that we do not rescale the horizon radius to one, as in $[15,16]$ - the physics in the presence of a scalar field is unambiguous if we do not use this rescaling.

\section{$2.1 \quad$ The model}

We will focus on a 5-dimensional theory of gravity coupled to a massless scalar and an abelian electromagnetic field whose action is ${ }^{4}$

$$
\begin{aligned}
S_{\mathrm{EM}} & =\frac{1}{16 \pi G_{5}} \int d^{5} x \sqrt{-g}\left(R+12-e^{\alpha \varphi(r)} F_{\mu \nu} F^{\mu \nu}-\partial_{\mu} \varphi \partial^{\mu} \varphi\right)+S_{\mathrm{CS}} \\
S_{\mathrm{CS}} & =\frac{\frac{\zeta}{3}}{16 \pi G_{5}} \int d^{5} x \epsilon^{\mu \nu \rho \sigma \gamma} A_{\mu} F_{\nu \rho} F_{\sigma \gamma} .
\end{aligned}
$$

In this paper, we consider a constant moduli potential, $V(\phi)=2 \Lambda=-12 / l^{2}$, and also fix the radius of AdS to be $l=1$.

Since the equations of motion for the gauge field simplify, we choose the constant in front of the Chern-Simons term to be $\zeta / 3$. The action (2.1) with various values for $\alpha$ resembles (truncated) actions obtained in string compactifications [20-23]. The coupling $\zeta$ captures the strength of the anomaly of the boundary current.

The equations of motion for the metric, scalar, and electromagnetic field $\left(F_{\mu \nu}=\partial_{\mu} A_{\nu}-\right.$ $\left.\partial_{\nu} A_{\mu}\right)$ are

$$
\begin{aligned}
R_{\mu \nu}+4 g_{\mu \nu}+e^{\alpha \varphi(r)}\left(\frac{1}{3} F^{2} g_{\mu \nu}-2 F_{\mu \rho} F_{\nu \sigma} g^{\rho \sigma}\right)-\partial_{\mu} \varphi \partial_{\nu} \varphi & =0 \\
\frac{1}{\sqrt{-g}} \partial_{\mu}\left(\sqrt{-g} \partial^{\mu} \varphi\right) & =-\frac{1}{2} \alpha e^{\alpha \varphi(r)} F_{\mu \nu} F^{\mu \nu}, \\
e^{\alpha \varphi(r)} \partial_{\nu}\left(\sqrt{-g} F^{\nu \mu}\right)+\frac{\zeta}{4} \epsilon^{\mu \rho \sigma \gamma \delta} F_{\rho \sigma} F_{\gamma \delta} & =0,
\end{aligned}
$$

where we have varied the scalar and the electromagnetic field independently. The Bianchi identities for the gauge field are $F_{[\mu \nu ; \lambda]}=0$.

Since we are interested in a theory for which the Chern-Simons term has a non-trivial contribution, we consider the following ansatz for the gauge field: ${ }^{5}$

$$
A=E(r) d t-\frac{B}{2} y d x+\frac{B}{2} x d y-P(r) d z
$$

The magnetic field, $B$, is fixed to be constant by the Bianchi identities. Thus, the field

\footnotetext{
${ }^{4}$ We also have to add counterterms to regularize the action — for (2.1), the counterterms are given in [38] (see, also, the nice review [39] and references therein for a more detailed discussion).

${ }^{5}$ Please note that our $P^{\prime}(r)$ is the same as $P(r)$ in $[15,16]$ and our $Z(r)$ in the ansatz of the metric $(2.8)$ is their $C(r)$.
} 
strength is ${ }^{6}$

$$
F=\left(\begin{array}{ccccc}
0 & E^{\prime}(r) & 0 & 0 & -P^{\prime}(r) \\
-E^{\prime}(r) & 0 & 0 & 0 & 0 \\
0 & 0 & 0 & B & 0 \\
0 & 0 & -B & 0 & 0 \\
P^{\prime}(r) & 0 & 0 & 0 & 0
\end{array}\right)
$$

where' denotes derivatives with respect to $r$.

Our analysis is on time-independent black hole solutions and so we consider the following ansatz for the metric

$$
d s^{2}=\frac{d r^{2}}{U(r)}-U(r) d t^{2}+e^{2 V(r)}\left(d x^{2}+d y^{2}\right)+e^{2 W(r)}(d z+Z(r) d t)^{2},
$$

which is compatible with the symmetries of the problem.

In this case, the horizon is located at (the biggest root of) $U\left(r_{h}\right)=0$ and the temperature can be easily computed on the Euclidean section — we obtain

$$
T=\frac{U^{\prime}\left(r_{h}\right)}{4 \pi} .
$$

By using the metric ansatz (2.8), we can rewrite the Maxwell equations as

$$
\begin{aligned}
{\left[Q(r) e^{2 V(r)+W(r)+\alpha \varphi(r)}\right]^{\prime}-2 \zeta B P^{\prime}(r) } & =0, \\
{\left[e^{2 V(r)+W(r)+\alpha \varphi(r)}\left(U(r) e^{-2 W(r)} P^{\prime}(r)-Q(r) Z(r)\right)\right]^{\prime}-2 \zeta B E^{\prime}(r) } & =0
\end{aligned}
$$

where

$$
Q(r)=E^{\prime}(r)+Z(r) P^{\prime}(r) .
$$

It is easier to work with combinations of Einstein equations rather than using directly (2.3). First, we extract the expressions of second derivatives of the functions that characterize the metric in the following way: we obtain $W^{\prime \prime}(r)$ from $(r r)-, V^{\prime \prime}(r)$ from $(x x)$ - , and $U^{\prime \prime}(r)$ from $(z z)$-component of Einstein equations.

Let us now consider the $(t z)$-component of Einstein equations in which we replace $W^{\prime \prime}(r), V^{\prime \prime}(r)$, and $U^{\prime \prime}(r)$ - we obtain

$$
e^{2 W(r)}\left[2 V^{\prime}(r) Z^{\prime}(r)+3 W^{\prime}(r) Z^{\prime}(r)+Z^{\prime \prime}(r)\right]-4 Q(r) P^{\prime}(r) e^{\alpha \varphi(r)}=0 .
$$

An important observation, which can be drawn by studying the system of equations (2.10)-(2.13), is that a non-zero magnetic field is not compatible with a constant function $Z(r)$ (and, also, $P(r)$ ).

The other (independent) combinations of Einstein equations are obtained as follows: by replacing $U^{\prime \prime}(r)$ in the $(r r)$-component of Einstein equations we get

$$
\begin{aligned}
& 2 B^{2} e^{\alpha \varphi(r)-4 V(r)}+2 Q(r)^{2} e^{\alpha \varphi(r)}+U(r)\left[2 V^{\prime}(r)+W^{\prime}(r)\right]^{2} \\
& +\left[U(r)\left(2 V^{\prime}(r)+W^{\prime}(r)\right)\right]^{\prime}+\frac{1}{2} e^{2 W(r)} Z^{\prime}(r)^{2}-12=0
\end{aligned}
$$

\footnotetext{
${ }^{6}$ Our convention for the coordinates is $(r, t, x, y, z)$.
} 
by replacing $W^{\prime \prime}(r)$ in the $(z z)$-component of Einstein equations

$$
\begin{aligned}
-4 Q(r)^{2} e^{\alpha \varphi(r)} & +U^{\prime \prime}(r)+U^{\prime}(r)\left[2 V^{\prime}(r)-W^{\prime}(r)\right]-2 e^{2 W(r)} Z^{\prime}(r)^{2} \\
+ & 2 U(r)\left[2 V^{\prime \prime}(r)-2 V^{\prime}(r) W^{\prime}(r)+2 V^{\prime}(r)^{2}+\varphi^{\prime}(r)^{2}\right]=0
\end{aligned}
$$

and the last one is, in fact, the $(x x)$-component of Einstein equations

$$
\begin{aligned}
e^{2 W(r)}[- & \left.4 B^{2} e^{\alpha \varphi(r)}-3 e^{4 V(r)} U^{\prime}(r) V^{\prime}(r)+12 e^{4 V(r)}\right]-2 Q(r)^{2} e^{4 V(r)+2 W(r)+\alpha \varphi(r)} \\
& +U(r) e^{4 V(r)}\left[2 P^{\prime}(r)^{2} e^{\alpha \varphi(r)}-3 e^{2 W(r)}\left(V^{\prime \prime}(r)+V^{\prime}(r) W^{\prime}(r)+2 V^{\prime}(r)^{2}\right)\right]=0 .
\end{aligned}
$$

We also use the ansatz of the metric in the equation of motion for the scalar (2.4) and so this equation becomes

$$
\left[U(r) e^{2 V(r)+W(r)} \varphi^{\prime}(r)\right]^{\prime}+\alpha e^{2 V(r)+W(r)+\alpha \varphi(r)}\left[B^{2} e^{-4 V(r)}+U(r) e^{-2 W(r)} P^{\prime}(r)^{2}-Q(r)^{2}\right]=0 .
$$

Due to the non-trivial coupling between the scalar and gauge fields, the equation (2.4) has a non-trivial right hand side. The non-vanishing electromagnetic field may also be understood as a source for the scalar field. Thus, the scalar charge is determined by the electric and magnetic charges and so it is not an independent parameter that characterizes the system - this charge plays an important role when the asymptotic value of the scalar is not fixed (see [41, 42]).

We would like to conclude this section with an observation on the Hamiltonian constraint. A vanishing Hamiltonian is a characteristic feature of any theory that is invariant under arbitrary coordinate transformations - for our system, we can obtain a first order differential equation by replacing $W^{\prime \prime}(r), V^{\prime \prime}(r)$, and $U^{\prime \prime}(r)$ in the $(t t)$-component of Einstein equations.

The Hamiltonian constraint, which can be enforced as an initial condition, has the following expression:

$$
\begin{aligned}
& 2 B^{2} e^{\alpha \varphi(r)-4 V(r)}+U(r)\left[-2 P^{\prime}(r)^{2} e^{\alpha \varphi(r)-2 W(r)}+4 V^{\prime}(r) W^{\prime}(r)+2 V^{\prime}(r)^{2}-\right. \\
& \left.-\varphi^{\prime}(r)^{2}\right]+2 Q(r)^{2} e^{\alpha \varphi(r)}+2 U^{\prime}(r) V^{\prime}(r)+U^{\prime}(r) W^{\prime}(r)+\frac{1}{2} e^{2 W(r)} Z^{\prime}(r)^{2}-12=0 .
\end{aligned}
$$

\subsection{Holographic data}

The AdS/CFT correspondence [3] is a concrete example of open/closed string duality. Remarkably, at strong coupling $\mathcal{N}=4 \mathrm{SYM}$ is best described by classical supergravity in this section, we closely follow the reviews [39, 43].

The observables in the field theory side of the duality are the correlation functions of gauge invariant operators, which are composites of the elementary fields. Any supergravity field, $\Phi$, corresponds to an operator, $\mathcal{O}$, in the (boundary) field theory - in particular, bulk gauge fields correspond to boundary symmetry currents.

We are interested in planar black hole solutions in AdS and so we will consider the topology of the AdS timelike boundary to be $R^{3} \times R^{+}$- the intrinsic coordinates of the boundary are denoted by $x$ and the radial coordinate by $r$. A concrete prescription to do computations on the Euclidean section was given in $[44,45]$. That is, the on-shell 
bulk partition function with the boundary conditions $\left.\Phi(x, r)\right|_{\infty}=\Phi_{0}(x)$ is the generating functional of the boundary QFT correlation functions:

$$
Z_{\mathrm{SUGRA}}\left[\Phi_{0}(x)\right]=\int\left\langle e^{\int d^{4} x \Phi_{0}(x) \mathcal{O}(x)}\right\rangle_{\mathrm{QFT}} .
$$

We work in the saddle point approximation and so the generating function of QFT is determined by the on-shell supergravity action, $W_{Q F T}\left[\Phi_{0}\right]=-S_{\text {onshell }}\left[\Phi_{0}\right]$. To compute the two-point function of a particular operator in the field theory, we should first solve the linearized equation of the corresponding supergravity field with the appropriate boundary conditions, then evaluate the on-shell sugra action, and, finally, compute the second functional derivative with respect to the source:

$$
<O(x) O(y)>=-\left.\frac{\delta^{2} S_{\text {onshell }}}{\delta \Phi_{0}(x) \delta \Phi_{0}(y)}\right|_{\Phi_{0}=0} .
$$

We are interested in a bulk gravity theory with an electromagnetic field non-minimally coupled to a scalar. In general there are two types of perturbations of AdS: those that modify the bulk but preserve the AdS asymptotics (e.g., a black hole is interpreted as a finite temperature state in dual QFT) and those that do not modify the bulk and act as sources for operators in the dual QFT. The AdS metric (graviton) couples to the stress tensor and the gauge field $A_{\mu}$ couples to the R-charged current $J^{\mu}$ of the field theory.

To obtain the viscosity (conductivity) we should compute the two-point functions of the stress tensor (current). Due to the coupling of the gauge field with a scalar, there will be a non-trivial 3-point function mixing two currents with a scalar operator - in this work, we are not interested in this correlation function.

The bulk solution is a geometry with a horizon (at zero or finite temperature) and to get the information about the hydrodynamics one should compute the retarded Green functions directly on the Lorentzian section. One reason is that, on the Euclidean section, there is a discrete set of frequencies, the Matsubara frequencies $(\omega=2 \pi i n T$ for bosons and $\omega=i \pi T(2 n+1) / 2$ for fermions). However, since we are interested in a small $\omega$ in the hydrodynamic approximation, an analytic continuation from the Euclidean section where there is a minimum frequency $\left(\omega_{m}=2 \pi i T\right)$ is not useful.

The general prescription for real-time retarded Green's functions within AdS/CFT duality can be found in $[46,47]$. The existence of hydrodynamics modes is reflected by the existence of the poles of the retarded correlators. However, to compute the viscosity bound, we will use a different prescription, namely the membrane paradigm proposal of [24].

We would like to present now a discussion on the boundary conditions for gauge fields. The bulk gauge field (2.7) is invariant under translations in the boundary coordinates $(x)$. The component tangent to the boundary of the gauge field is constant at large $r$, which corresponds to a magnetic field $B_{z}$ perpendicular on the (xy)-plane.

If there is an electric field, $E_{x}$, in $x$-direction, then the effect of a magnetic field in $z$ direction induces a current along $y$-direction. For, $A_{t}(x) \neq 0$ and $A_{i}=A_{y}(x) \neq 0$, the conserved currents on the boundary are the charge density, $q$, and the current density, $J$ 
— they are defined as

$$
q=\left.\frac{\delta S}{\delta A_{t}}\right|_{\text {boundary }}, \quad J^{i}=\left.\frac{\delta S}{\delta A_{i}}\right|_{\text {boundary }}
$$

To obtain the boundary current, we have to impose the following boundary conditions:

$$
\left.\delta A_{t}\right|_{\text {boundary }}=0,\left.\quad \delta A_{i}\right|_{\text {boundary }} \text { free. }
$$

The condition of regularity of the gauge potential at the horizon requires that $A_{t}$ should vanish (on the Euclidean section, the radius of the Euclidean time shrinks to zero at the horizon). Therefore, we should add a gauge term, $\Upsilon$ so that $A_{t}\left(r_{h}\right)-\Upsilon=0$. This term, which remains constant at the boundary, plays the role of a chemical potential for the electric charge.

Let us end up this section with a comment about the Chern-Simons term. The coupling of the gauge Chern-Simons term is proportional to the chiral anomaly of the corresponding current in the dual field theory. The current anomaly is given by a one-loop triangle Feynman diagram to the 3-point function of (R-)currents. Therefore, there also exists a chiral magnetic current which is induced parallel to the applied magnetic field, $J^{z} \sim \zeta B \Upsilon$.

\section{$3 \quad$ Near horizon geometry}

Shear viscosity of the boundary fluid can be computed from the knowledge of the near horizon physics only [24] - in section 4, we will present a detailed analysis. This observation is very useful when the bulk Lagrangian is very complicated. For example, for the model we are interested in, gravity is coupled to various matter fields in a non-trivial way and it is technically difficult to find an analytic solution of the system.

However, it is not a very complicated problem to find the near horizon geometry, i.e. how the metric and other fields behave in the near horizon limit. The reason is that, in principle, we do not have to solve any differential equation to find the near horizon geometry. A study of the extremal near horizon geometries is interesting in its own because it provides information about the instabilities that may appear in the theory.

The algorithm is as follows: first we obtain the field equations. Then, we consider a suitable near horizon ansatz for different fields - all fields should be regular at the horizon. Substituting the ansätze for fields in the corresponding equations of motion, one can solve them consistently order by order in $\left(r-r_{h}\right)$.

In this section, we will first find the near-horizon geometry of the non-extremal black hole, which we will need to compute the shear viscosity to entropy density ratio in section 4 . In the extremal limit, we will see that, due to the attractor mechanism, the near horizon geometry is universal regardless of the asymptotic values of the scalars. We present a detailed analysis of the branches of solutions with finite horizon area. 


\subsection{Non-extremal case}

As in $[15,16]$, we work with a coordinate system in which the solution takes the canonical form at the horizon. That is, the field strength $F_{H}$ and the metric $d s_{H}^{2}$ are

$$
\begin{aligned}
F_{H} & =q d r \wedge d t+B d x \wedge d y-p d r \wedge d z, \\
d s_{H}^{2} & =r_{H}^{2}\left(d x^{2}+d y^{2}+d z^{2}\right)
\end{aligned}
$$

where $q$ and $B$ are the charge density (of the black brane) and the magnetic field at the horizon, respectively. In this way, the gauge freedom is removed and the initial conditions are

$$
U\left(r_{h}\right)=Z\left(r_{h}\right)=P\left(r_{h}\right)=0, \quad V\left(r_{h}\right)=W\left(r_{h}\right)=\ln \left(r_{h}\right)
$$

A similar analysis (and numerical solutions) in the presence of the Gauss-Bonnet term but without the Chern-Simons term was presented in [48].

The generic solutions have a non-degenerate horizon. Near the event horizon, they admit a power series expansion of the form (using the definition of the temperature (2.9) in the expression of $U$ ):

$$
\begin{aligned}
U(r) & =4 \pi T\left(r-r_{h}\right)+u_{2}\left(r-r_{h}\right)^{2}+\cdots, \\
V(r) & =\ln \left(r_{h}\right)+v_{1}\left(r-r_{h}\right)+v_{2}\left(r-r_{h}\right)^{2}+\cdots, \\
W(r) & =\ln \left(r_{h}\right)+w_{1}\left(r-r_{h}\right)+w_{2}\left(r-r_{h}\right)^{2}+\cdots, \\
Z(r) & =z_{1}\left(r-r_{h}\right)+z_{2}\left(r-r_{h}\right)^{2}+\cdots, \\
E(r) & =q\left(r-r_{h}\right)+q_{1}\left(r-r_{h}\right)^{2}+q_{2}\left(r-r_{h}\right)^{3}+\cdots, \\
P(r) & =p\left(r-r_{h}\right)+p_{1}\left(r-r_{h}\right)^{2}+\cdots, \\
\varphi(r) & =\varphi_{h}+\varphi_{1}\left(r-r_{h}\right)+\varphi_{2}\left(r-r_{h}\right)^{2}+\cdots
\end{aligned}
$$

It is important to emphasize that, what is generally called near horizon geometry for a non-extremal black hole is just a truncation of the above series expansion. To compute the shear viscosity, though, we need also some data at the order $\left(r-r_{h}\right)^{2}$.

Another observation is that, in principle, one can use a boost transformation in $z$ direction to set $p=0$ (see $[15,16]$ ). However, the boost transformation is singular at some point outside the black hole horizon. In our analysis we keep the value of $p$ non-zero and determine it in terms of other horizon data. We will see in section 4 that the expressions for the entropy, shear viscosity, and their ratio remain unchanged if we set the horizon value of $P^{\prime}(r)$ to be zero (in other words, they do not depend of $p$ ). This is expected due to the fact that the physical quantities should be invariant under the boost transformations.

By substituting the ansatz (3.3) in the field equations, we get the following expressions 
for the coefficients at the order $\left(r-r_{h}\right):^{7}$

$$
\begin{aligned}
v_{1}= & -\frac{2 B^{2} e^{\alpha \varphi_{h}}+r_{h}^{4}\left(q^{2} e^{\alpha \varphi_{h}}-6\right)}{6 \pi T r_{h}^{4}}, \\
w_{1}= & \frac{4 B^{2} e^{\alpha \varphi_{h}}-r_{h}^{4}\left(4 q^{2} e^{\alpha \varphi_{h}}+3 z_{1}^{2} r_{h}^{2}-24\right)}{24 \pi T r_{h}^{4}}, \\
p= & \frac{q\left(2 B \zeta e^{-\alpha \varphi_{h}}+z_{1} r_{h}^{3}\right)}{4 \pi T r_{h}}, \\
\varphi_{1}= & \frac{\alpha e^{\alpha \varphi_{h}}\left(q^{2} r_{h}^{4}-B^{2}\right)}{4 \pi T r_{h}^{4}}, \\
q_{1}= & \frac{e^{-2 \alpha \varphi_{h}}}{16 \pi T r_{h}^{4}}\left(2 B^{2}\left[\left(\alpha^{2}+2\right) e^{3 \alpha \varphi_{h}}+4 \zeta^{2}\right]\right. \\
& \left.-r_{h}^{4} e^{2 \alpha \varphi_{h}}\left[2 q^{2}\left(\alpha^{2}-2\right) e^{\alpha \varphi_{h}}+z_{1}^{2} r_{h}^{2}+24\right]\right) .
\end{aligned}
$$

However, in higher derivative gravity theories the only coefficient at the order $\left(r-r_{h}\right)^{2}$, which we need for viscosity bound computation, is $u_{2}$. But, for completeness, we present the expressions of all the other coefficients that appear at order $\left(r-r_{h}\right)^{2}$, in appendix B.

A non-extremal charged scalar black hole is characterized by four independent parameters: the mass, electric charge, magnetic field, and also the value of the scalar at the horizon, $\varphi_{h}$. In this case, the horizon radius (and so the entropy) and the horizon value of the scalar depend of the asymptotic boundary data $\left(\varphi_{\infty}\right)$. We will see in the next subsection that this is in contrast with the extremal case for which we obtain an attractor behaviour at the horizon.

At first sight, it may seem surprising that the data (3.4), (B.1) we need to compute the entropy and shear viscosity depend also on $z_{1}$, a coefficient that we do not compute explicitly. However, we will see in section 4 that the final values of the physical quantities depend in fact just on four independent parameters, namely $\left(q, B, r_{h}, \varphi_{h}\right)$ - we 'trade' the mass for the horizon radius and so the independent parameters that completely characterize the black hole are the ones mentioned above.

\subsection{Extremal limit and attractor mechanism}

It is by now well known that the extremal black holes in theories of Einstein gravity with scalar fields non-trivially coupled with abelian gauge fields have an enhanced symmetry of the near horizon geometry $[49,50]$. That is, the near horizon geometry contains an $A d S_{2}$ spacetime.

The attractor mechanism [51] is also valid for non-BPS extremal black holes [52-54 $]^{8}$ a qualitative explanation is that, due to the infinite long throat of $A d S_{2}$, the near horizon geometry has no memory of asymptotic data. Consequently, the near horizon geometry is universal and the entropy does not depend on the asymptotic values of the scalars.

\footnotetext{
${ }^{7}$ We obtain the results as functions of the coefficients $\left(T, q, B, \varphi_{h}, r_{h}\right.$, and $\left.z_{1}\right)$ - this will simplify the computations of the shear viscosity.

${ }^{8} \mathrm{~A}$ generalization to stationary non-susy black holes was given in [55]. More details on the nonsupersymmetric attractors can be found in the nice reviews [56-60].
} 
In this section we present a careful analysis of the extremal near horizon geometry by using directly the equations of motion and also the entropy function formalism. The advantage of the latter is that we can compute the physical charges and obtain analytic expressions for the entropy density. In this way, we can confirm the numerical analysis of [17].

The (non-supersymmetric) attractor mechanism in AdS and its embedding in string theory were studied in [61]. In the rest of this section we follow closely [48, 61], though we should keep in mind that our goal is not to investigate just the flow of the entropy but also the flow of some hydrodynamic transport coefficients, and we also consider a non-trivial Chern-Simons term.

\subsubsection{Attractor mechanism}

In AdS spacetime, the BPS condition is different than the extremal limit. For static black holes, the BPS limit is a naked singularity, but in the extremal limit the entropy can remain finite.

Unlike the non-extremal case, in the extremal case the near horizon geometry is an exact solution of the equations of motion and not a truncation in a Taylor expansion. Let us consider the most general ansatz for the near horizon geometry:

$$
\begin{aligned}
d s^{2} & =L\left(\frac{d r^{2}}{r^{2}}-r^{2} d t^{2}\right)+v_{1}\left(d x^{2}+d y^{2}\right)+v_{2}\left(d z+z_{1} r d t\right)^{2}, \\
F & =q d r \wedge d t+B d x \wedge d y \\
\varphi(r) & =\varphi_{h}
\end{aligned}
$$

Another important difference with the non-extremal case is that, due to the enhanced symmetry of the near horizon geometry, $p$ vanishes (the $z$ component of the gauge field is constant). As a consistency check, it can be shown that with this ansatz, in the absence of the scalar field, we obtain the results of $[15,16] .^{9}$

Let us consider now in more detail the non-trivial case when the scalar is turned on. The first Maxwell equation can be easily integrated and we obtain

$$
Q_{p}=\frac{v_{1}^{2} v_{2} q^{2} e^{2 \alpha \varphi_{h}}}{L^{2}}
$$

where the integration constant is related to the physical charge. ${ }^{10}$

\footnotetext{
${ }^{9}$ We emphasize again that the authors of $[15,16]$ have shifted the radial coordinate, $r$, to set the horizon at $r_{h}=1$.

${ }^{10} Q_{p}$ includes the contribution from constant $z$ component of gauge field.
} 
We use the following system of independent equations: ${ }^{11}$

$$
\begin{aligned}
4 B^{2} L^{2} v_{2} e^{2 \alpha \varphi_{h}}+v_{1}^{2} v_{2} e^{\alpha \varphi_{h}}\left(24 L^{2}-6 L+3 v_{2} z_{1}^{2}\right)+8 L^{2} Q_{p}^{2} & =0 \\
4 B^{2} L^{2} v_{2} e^{2 \alpha \varphi_{h}}-4 L^{2}\left(Q_{p}^{2}-6 v_{1}^{2} v_{2} e^{\alpha \varphi_{h}}\right)-3 v_{1}^{2} v_{2}^{2} z_{1}^{2} e^{\alpha \varphi_{h}} & =0, \\
-2 B^{2} e^{\alpha \varphi_{h}}-\frac{Q_{p}^{2} e^{-\alpha \varphi_{h}}}{v_{2}}+6 v_{1}^{2} & =0 \\
v_{2}\left(\frac{4 B^{2} e^{\alpha \varphi_{h}}}{v_{1}^{2}}+24\right)-\frac{4 Q_{p}^{2} e^{-\alpha \varphi_{h}}}{v_{1}^{2}}-\frac{3 v_{2}^{2} z_{1}^{2}}{L^{2}} & =0 \\
\frac{2 B^{2} v_{2} e^{\alpha \varphi_{h}}}{v_{1}}-\frac{v_{1} v_{2}^{2} z_{1}^{2}}{2 L^{2}} & =0, \\
Q_{p}\left(\frac{8 B L \zeta e^{-\alpha \varphi_{h}}}{v_{1} \sqrt{v_{2}}}+4 z_{1}\right) & =0, \\
\alpha\left(B^{2} L^{2}-\frac{L^{2} Q_{p}^{2} e^{-2 \alpha \varphi_{h}}}{v_{2}}\right) & =0 .
\end{aligned}
$$

As we already explained above, we expect that the near horizon geometry to be completely fixed by the charges. By solving the system of equations (3.7), we obtain the following expressions for $A d S_{2}$ radius, size of transverse space, strength of fibration, and horizon value of the scalar:

$$
L=\frac{1}{12}, \quad v_{1}=\frac{B \zeta^{1 / 3}}{\sqrt{2}}, \quad v_{2}=\left(\frac{Q_{p}}{B \zeta^{2 / 3}}\right)^{2}, \quad z_{1}=-\frac{B \zeta^{2 / 3}}{3 \sqrt{2} Q_{p}}, \quad \varphi_{h}=\frac{2 \ln (\zeta)}{3 \alpha} .
$$

The radius of $A d S_{2}$ is the same as the radius of $A d S_{5}$. This is due to the fact that we work with planar black holes - for extremal spherical black holes in $A d S_{5}$, the radius of $A d S_{2}$ does not match the radius of $A d S_{5}$ [61].

One important observation is that the horizon value of the scalar is fixed by the ChernSimons coupling. We will reobtain this result by using the entropy function.

For a regular extremal solution whose entropy does not vanish, there is a large ground state degeneracy. However, an important question is then, if the entropy is 'stable' against changes of the boundary conditions for fields in the bulk. In $[15,16]$, the authors argue that the near horizon geometry $\left(A d S_{2} \times R^{3}\right)$ of the purely electrically charged brane is unstable under the addition of a magnetic field.

In the rest of this section we argue that, in the presence of the scalar and electromagnetic field, the entropy does not change under general perturbations of the scalar. A similar discussion for a 5-dimensional theory without the Chern-Simons term can be found in $[48,61] .{ }^{12}$

The arguments are as follows. Due to the non-trivial coupling between the scalar and gauge fields, there is an 'effective potential' for the scalar [54]. If the effective potential

\footnotetext{
${ }^{11}$ The first one is the $(r r)$-component of Einstein equation, the second equation is a combination of $(r r)$ and $(t t)$-components, the third one is the $(x x)$-component, the fourth one is the $(t z)$-component, the next one is a combination of $(x x)$ - and $(z z)$-components, and the last two are the remaining non-trivial Maxwell equation and the scalar equation, respectively.

${ }^{12}$ Recently, the authors of [62] have used the same kind of arguments to discuss charged scalar black holes in $A d S_{4}$.
} 
has a stable minimum at the horizon, the solution is regular and the entropy is determined completely by the charge parameters (if there are flat directions, not all moduli are stabilized, but the entropy does not depend of the flat directions).

We can see that, without a gauge field, the theory is scaling invariant and so the scalar field is always massless. By turning on just one gauge field (electric or magnetic) the symmetry is broken and one expects that the solution is not regular. The reason is that, even if an effective potential is generated, the potential has no stable minimum.

However, by turning on a second gauge field (in our case, we consider an electromagnetic field) we expect to obtain regular solutions. The effective potential can have stable minima and the near horizon geometry is universal.

One important observation, though, is that the ansatz of our solution is different than the one of $[54]$ — we have non-trivial terms $(d z d t)$ in the metric. A concrete expression for the effective potential in this case is not known. However, in the near horizon limit the effective potential method should be equivalent with the entropy function method of Sen. In what follows, we will use the attractor equations to find the near horizon geometry (and so the entropy density) of the extremal solutions.

\subsubsection{Entropy function}

By using the entropy function formalism we can explicitly compute the physical charges and obtain an analytic expression for the entropy density. Since our theory contains the gauge Chern-Simons term, which is not gauge invariant, we have to Kaluza-Klein (KK) reduce our action to four dimensions.

Let us consider first the case with the scalar turned off. We consider the $z$ direction compactified on a circle of radius $\beta$ and a general KK ansatz

$$
\begin{aligned}
g_{\alpha \beta} d x^{\alpha} d x^{\beta} & =G_{a b} d x^{a} d x^{b}+G_{A B}\left(d y^{A}+\bar{A}_{a}^{A} d x^{a}\right)\left(d y^{B}+\bar{A}_{a}^{B} d x^{a}\right), \\
A^{(5)} & =A_{\mu}^{(5)} d x^{\mu}=A_{a}^{(4)} d x^{a}+C_{B}\left(x^{a}\right)\left(d y^{B}+\bar{A}_{a}^{B} d x^{a}\right),
\end{aligned}
$$

where $a, b$ are $4 \mathrm{D}$ indices and $A, B$ are compact indices ( $z$ in our case). With this notation we have splitted the coordinates as $x^{\mu}=\left(x^{a}, y^{A}\right)$ and so $A_{\mu}^{(5)}$ is the 5-dimensional gauge potential, $A_{a}^{(4)}$ is the 4-dimensional gauge potential, and $\bar{A}_{a}^{B}$ is the KK gauge potential.

We use the following results of the dimensional reduction [40] (there is no dependence of KK coordinates):

$$
\begin{aligned}
\sqrt{-g}= & \sqrt{-G} \sqrt{\operatorname{det}\left(G_{A B}\right)}, \quad F^{(5) 2}=F^{(5) \mu \nu} F_{\mu \nu}^{(5)}=F^{(5) a b} F_{a b}^{(5)}+2 F^{(5) a B} F_{a B}^{(5)} \\
R_{5}= & R_{4}-\frac{1}{4} G^{a c} G^{b d} G_{A B} \bar{F}_{a b}^{A} \bar{F}_{c d}^{B}+\frac{1}{4} \partial_{a} G_{A B} \partial^{a} G^{A B}-\frac{1}{4} G^{A B} \partial_{a} G_{A B} G^{C D} \partial^{a} G_{C D}- \\
& -\partial_{a}\left(G_{A B} \partial^{a} G_{A B}\right) .
\end{aligned}
$$

Now, we are ready to write down the ansatz we are interested in - the metric, gauge field, and relations between the 5-dimensional gauge potential, KK gauge potential, and 
4-dimensional gauge potential are

$$
\begin{aligned}
d s^{2} & =L\left(-\frac{d r^{2}}{r^{2}}+r^{2} d t^{2}\right)+v_{1}\left(d x^{2}+d y^{2}\right)+v_{2}\left(d z+z_{1} r d t\right)^{2}, \\
A_{\mu}^{(5)} d x^{\mu} & =\vartheta r d t-\frac{B}{2} y d x+\frac{B}{2} x d y-p_{1}\left(d z+z_{1} r d t\right), \\
F_{r t}^{(5)} & =q=\vartheta-p_{1} z_{1}, \quad F_{x y}^{(5)}=F_{x y}^{(4)}=B, \quad F_{r t}^{(4)}=\vartheta, \quad \bar{F}_{r t}^{z}=z_{1} .
\end{aligned}
$$

The on-shell action and entropy function are

$$
\begin{aligned}
& S=\frac{\mathcal{A}_{x y} \beta}{16 \pi G_{5}}\left[L \sqrt{v_{1}^{2} v_{2}}\left(-\frac{2 B^{2}}{v_{1}^{2}}+\frac{2\left(\vartheta-p_{1} z_{1}\right)^{2}}{L^{2}}+\frac{v_{2} z_{1}^{2}-4 L}{2 L^{2}}+12\right)+8 B p_{1} \zeta\left(\frac{p_{1} z_{1}}{2}-\vartheta\right)\right], \\
& \mathcal{E}=2 \pi \beta \mathcal{A}_{x y}\left(Q \vartheta+\Theta z_{1}-\frac{S}{\beta \mathcal{A}_{x y}}\right)
\end{aligned}
$$

where $Q$ is the 4-dimensional physical charge, $\Theta$ is the physical charge associated to $K K$ gauge field, and $\mathcal{A}_{x y}=\int d x d y$.

The equations of motion in the near horizon limit are

$$
\begin{aligned}
-\frac{\beta \pi \sqrt{v_{2}}}{L v_{1}^{2}}\left[4 B^{2} L^{2}+v_{1}^{2}\left(-4 L+24 L^{2}+4 q^{2}+v_{2} z_{1}^{2}\right)\right] & =0, \\
\frac{\beta \pi}{L v_{1} \sqrt{v_{2}}}\left[4 B^{2} L^{2}-v_{1}^{2}\left(-4 L+24 L^{2}+4 q^{2}+3 v_{2} z_{1}^{2}\right)\right] & =0, \\
\frac{\beta \pi \sqrt{v_{2}}}{L v_{1}^{2}}\left[4 B^{2} L^{2}+v_{1}^{2}\left(-24 L^{2}+4 q^{2}+v_{2} z_{1}^{2}\right)\right] & =0 \\
\frac{2 \beta \pi}{L}\left[L\left(\Theta-4 B p_{1}^{2} \zeta\right)+v_{1} \sqrt{v_{2}}\left(4 p_{1} q-v_{2} z_{1}\right)\right] & =0, \\
\frac{8 \beta \pi q}{L}\left[2 B L \zeta+v_{1} \sqrt{v_{2}} z_{1}\right] & =0, \\
2 \pi \beta\left(8 B p_{1} \zeta-\frac{4 v_{1} \sqrt{v_{2}} q}{L}+Q\right) & =0 .
\end{aligned}
$$

First, we solve the last equation to obtain the 5-dimensional electric potential. Then, we use this result in all the other equations, and from the forth equation we obtain the following expression for the KK potential: ${ }^{13}$

$$
z_{1}=\frac{L\left(p_{1} Q+\Theta+4 B p_{1}^{2} \zeta\right)}{v_{1} v_{2}^{3 / 2}} .
$$

Next, we compute $p_{1}$ from the sum of the first two equations - we obtain a 4 th order equation with the following four solutions:

$$
\begin{array}{ll}
p 1=-\frac{Q+\sqrt{Q^{2}-16 B\left(2 B v_{2}+\Theta\right) \zeta}}{8 B \zeta}, & p 1=\frac{-Q+\sqrt{Q^{2}-16 B\left(2 B v_{2}+\Theta\right) \zeta}}{8 B \zeta} \\
p 1=\frac{-Q+\sqrt{Q^{2}+16 B\left(2 B v_{2}-\Theta\right) \zeta}}{8 B \zeta} & p 1=-\frac{Q+\sqrt{Q^{2}+16 B\left(2 B v_{2}-\Theta\right) \zeta}}{8 B \zeta} .
\end{array}
$$

\footnotetext{
${ }^{13}$ We note that by combining the first equations we get $L=1 / 12$.
} 
In the absence of the magnetic field, we expect a trivial fibration for our geometry: we can see that the physical solutions are the second and third ones because $p_{1}$ is finite in the limit $B \rightarrow 0$. Therefore, depending on $p_{1}$ solution, we have two distinct families of solutions. To compute the other near horizon parameters, we proceed as follows: by subtracting the third equation from the first one we solve for $v_{2}$, and get

$$
v_{2}=\frac{L\left(Q^{2}-16 B \Theta \zeta\right)}{8\left[v_{1}^{2}+4 B^{2} L(-1 \pm \zeta)\right]}
$$

The $+/-$ solution corresponds to the second and third solution for $p_{1}$, respectively.

The 5th equation, which is very important for understanding the solutions, and the entropy are

$$
B(1 \pm \zeta) \frac{\sqrt{3 v_{1}^{2}-B^{2}}}{36 \sqrt{2} v_{1}}=0, \quad S=\beta v_{1} \pi \sqrt{\frac{Q^{2}-16 B \Theta \zeta}{6 v_{1}^{2}+2 B^{2}(-1 \pm \zeta)}}
$$

It is now clear that, from the equation (3.16), there are three distinct branches: ${ }^{14}$

$$
B=0, \quad q=0\left(v_{1}^{2}=B^{2} / 3\right), \quad \zeta= \pm 1 .
$$

From (3.16), we can also read off the corresponding entropy for all three branches:

Branch 1: $B=0$

This branch corresponds to the usual Reissner-Nordström solution and its entropy is

$$
S=\frac{\beta \mathcal{A}_{x y} \pi Q}{\sqrt{6}} .
$$

Branch 2: $q=0\left(B / v_{1}=\sqrt{3}\right)$

We compute all near horizon parameters in terms of physical charges. We obtain $p_{1}=-\frac{Q}{8 B \zeta}$ and also find two possible solutions for $z_{1}$ and $v_{2}$ :

$$
\begin{array}{ll}
\text { set1 : } \quad \mathrm{z}_{1}=-\frac{2 \sqrt{2} \mathrm{~B}}{\sqrt{3} \sqrt{\frac{\mathrm{Q}^{2}}{\zeta}-16 \mathrm{~B} \Theta}}, \quad v_{2}=\frac{1}{32 \mathrm{~B}^{2}}\left(\frac{\mathrm{Q}^{2}}{\zeta}-16 \mathrm{~B} \Theta\right), \\
\text { set2 } \quad \mathrm{z}_{1}=\frac{2 \sqrt{2} \mathrm{~B}}{\sqrt{3} \sqrt{16 \mathrm{~B} \Theta-\frac{\mathrm{Q}^{2}}{\zeta}}}, \quad v_{2}=\frac{1}{32 \mathrm{~B}^{2}}\left(16 \mathrm{~B} \Theta-\frac{\mathrm{Q}^{2}}{\zeta}\right) .
\end{array}
$$

The corresponding entropies are

$$
S_{1}=\frac{\beta \mathcal{A}_{x y} \pi}{\sqrt{6}} \sqrt{\frac{Q^{2}}{\zeta}-16 B \Theta}, \quad, \quad S_{2}=\frac{\beta \mathcal{A}_{x y} \pi}{\sqrt{6}} \sqrt{16 B \Theta-\frac{Q^{2}}{\zeta}} .
$$

\footnotetext{
${ }^{14}$ We rewrite $q$ in terms of the physical parameters $q=\frac{\sqrt{3 v_{1}^{2}-B^{2}}}{6 \sqrt{2} v_{1}}$ to obtain the constraint between $v_{1}$ and $B$ for $q=0$.
} 
Branch 3: $\zeta= \pm 1$

In this case, one of the near horizon equations of motion, in fact, is used to get a fixed value for $\zeta$. Therefore, we can not compute all the parameters, one of them can not be fixed. However, as expected, the entropy depends only on the physical charges:

$$
S=\beta \mathcal{A}_{x y} \pi \sqrt{\frac{Q^{2} \mp 16 B \Theta}{6}} \quad \text { when } \zeta= \pm 1
$$

Now, we are ready to compare our analytic results with the numerical results of [17]. We just saw that with the entropy function formalism we get a similar condition as the one of [15-17], namely $q B(1 \pm \zeta)=0$. Therefore, we also obtain solutions for $q B=0$ or $\zeta= \pm 1$. In the first case, when $\zeta \neq \pm 1$, there is a regular solution (when $B=0$, branch 1 ) with the expected near horizon geometry $\left(A d S_{2} \times R^{3}\right)$.

For the case $q=0$ (branch 2), depending on the sign of $\zeta$, we have different situations. Let us assume that the physical $K K$ charge is $\Theta>0$. When $\zeta>0$ (but $\neq 1$ ), the positivity of $v_{2}$ (which is also reality of $z_{1}$ ) implies that $B$ has a critical value $B_{c}=\frac{Q^{2}}{16 \Theta \zeta}$ (solution set 1). Once $B$ crosses that critical value, the solution set 1 becomes singular and the solution set 2 takes over. In this case, for a generic value of the magnetic field, the near-horizon geometry is always $A d S_{3} \times R^{2}$ (see, also, $[15,16]$ ). For $\zeta<0$ (and $\neq-1$ ), the solution set 1 does not exist for any positive value of the magnetic field. In this case, the only possible solution is set 2, which is a regular (finite horizon area) solution for any positive value of $B$. We would also like to point out that, when the Chern-Simons coupling vanishes, it seems there is no finite area solution in the case $q=0$ and non-zero magnetic field.

In the second case, $\zeta= \pm 1$, there is a family of solutions for which $2\left(B / v_{1}\right)^{2}+(q / L)^{2}-$ $6=0$ (this constraint is obtained by solving the third and fifth equation in (3.13) and matches the condition of $[15,16]$ where the horizon radius is rescaled so that $\left.v_{1}=1\right)$. In this case for $\zeta=1$ there exists a critical magnetic field $B_{c}=\frac{Q^{2}}{16 \Theta}$ above which the solution becomes a naked singularity. For $\zeta=-1$ the solution exists for any value of $B$.

The near horizon geometry smoothly interpolates between $A d S_{2} \times R^{3}$ (for $B=0$ ) and $A d S_{3} \times R^{2}$ (for $q=0$ ) - in between, the near horizon geometry is in fact an warped $A d S_{3} \times R^{2} .15$

Let us know discuss the case when the modulus is turned on. The analysis is very similar with our computations above - we present the details in appendix C. Unlike in the previous section where we have solved the equations of motion in the near horizon geometry, by using the entropy function we can compute the physical charges in four dimensions. In five dimensions, due to the existence of the gauge Chern-Simons term, one has to be careful about the definition of the physical charge - we will present a detailed analysis of this aspect in the forthcoming work [18].

By using the attractor equations from appendix $\mathrm{C}$, we obtain the same horizon value of the modulus

$$
\varphi_{h}=\frac{2}{3 \alpha} \ln |\zeta|
$$

\footnotetext{
${ }^{15}$ The $A d S_{3}$ spacetime is a special fibration of $S^{1}$ over $A d S_{2}$ and the case $q=0$ corresponds to this special fibration.
} 
as in the previous subsection. In general, due to the attractor mechanism, in the extremal case the horizon values of the moduli are fixed by the charges. Interestingly enough, this is not the case here: the modulus is fixed by the Chern-Simons coupling alone. We will present in Discussion section an argument of why this is the case.

Another observation is that the physical magnetic field is also fixed by the ChernSimons coupling:

$$
\frac{B}{v_{1}}= \pm \sqrt{2} e^{-\alpha \varphi_{h} / 2}
$$

or, equivalently, the parameter $v_{1}$ is fixed only by the magnetic field parameter and the Chern-Simons coupling and does not depend on the electric field. This is due to the fact that we can rescale $x$ and $y$ coordinates in our solution to make $v_{1}=1$, and this rescaling affects just the magnetic field - that can be easily seen from our ansatz for the metric and gauge potential (3.11).

In appendix $\mathrm{C}$, we present a detailed computation of the entropy - the result is

$$
S=\beta \mathcal{A}_{x y} \pi \sqrt{\frac{Q^{2}-16 B \Theta \zeta}{6|\zeta|^{2 / 3}}} .
$$

It is not our goal to make a detailed analysis with the scalars turned on here (for different $\alpha$ and 'anisotropic near horizon geometries'), but we observe that, when just the electric or magnetic field is turned on, the solution is a naked singularity. This is similar with our discussion at the end of the previous subsection, when without the Chern-Simons term, we have argued that the effective potential does not have a minimum and so there is no regular solution [48, 61]. We do not know the form of the effective potential when the Chern-Simons term is present, but that can be easily seen from the equation of motion of the scalar (the last equation in (C.1) ). Consequently, there is just one branch of finite area solutions when the modulus is turned on.

As in the case with the scalar turned off, there also exists a critical value of the magnetic field for which the entropy shrinks to zero (for $\zeta>0$ ). So, the geometry is stable against scalar fluctuations but not against the magnetic field. However, this interpretation (and also the interpretations of [15-17]) should be taken with caution. It is expected that the higher derivative corrections will 'dress' the singularity with a horizon and so a more general analysis is important in this context.

\section{Shear viscosity to entropy density ratio}

For black objects with translation invariant horizon, for example black brane geometry, one can also discuss the hydrodynamics - long wave length deviation (low frequency fluctuation) from thermal equilibrium.

In addition to the thermodynamic quantities the black brane is also characterized by the hydrodynamic parameters like viscosity, diffusion constant, electrical conductivity, et cetera. The black D3-brane geometry with low energy fluctuations (i.e. with hydrodynamic behaviour) is dual to a finite temperature gauge theory plasma living on boundary with hydrodynamic fluctuations. 
In this section we will study the low frequency behaviour of boundary plasma dual to the gravity model presented in section 2. To do this, we use the membrane paradigm proposal of [24] and its generalization to higher derivative gravity theories [25, 26].

\subsection{Membrane paradigm and viscosity bound}

To an external observer, the black hole physics appears to be equivalent to the physics of a dynamical fluid membrane $[63,64]$. In other words, the black hole is equivalent with a set of surface charges and currents at the stretched horizon. ${ }^{16}$

In the Lorentzian prescription of [46] for computing the Green functions, one should impose in-falling boundary conditions for the fields at the horizon. However, when computing the two-point functions, just the contribution from the boundary has to be taken. An explanation of why the surface terms coming from the horizon must be dropped was given in [47] (see, also, [65-67]): the boundary conditions should be defined in Kruskal coordinates and one has to work with the full analytic extension of the black hole.

An important observation, which relates the work of [46] with the membrane paradigm, was made in [24] (see, also, [27, 68]). That is, the response functions in AdS/CFT are similar with the membrane response when the membrane is 'pushed' at the boundary. In this way, the membrane paradigm physical quantities are in fact concrete gauge theory observables. ${ }^{17}$

Interestingly, it was observed in [69] that only with the knowledge of the near horizon geometry one can easily calculate the shear viscosity of boundary fluid. One does not need to know the full analytic solutions of Einstein equations - this method is especially useful in higher derivative AdS gravity theories.

As a warm-up exercise, let us consider first the model presented in section 2.1. We apply the method of [24] to compute the shear viscosity coefficient of the boundary fluid for the action (2.1).

Let us consider a metric perturbation of the form:

$$
g_{x y}=g_{x y}^{(0)}+h_{x y}(r, x)=g_{x y}^{(0)}[1+\epsilon \Phi(r, x)] .
$$

At first sight, it seems that the dual gravitational mode (4.1) does not generally decouple. Interestingly enough, the decoupling occurs when the momentum vanishes and this is what we need for the computation of the viscosity in the hydrodynamics limit — we provide a detailed derivation of this claim in appendix D. ${ }^{18}$

By plugging (4.1) in the action and keeping the terms at order $\epsilon^{2}$ (at the first order in $\epsilon$, we obtain the equations of motion for gravitons), we get the following effective action

\footnotetext{
${ }^{16}$ The stretched horizon is a timelike surface just outside the true horizon. We emphasize that the charges and currents are fictitious in the sense that a falling observer through the stretched horizon does not detect any surface sources. However, for an external observer, their presence is consistent with all external field configurations.

${ }^{17}$ The infalling boundary conditions at the horizons are the regularity conditions in the membrane paradigm: near the horizon the fields depend trough a non-singular combination of $r$ and $t$, namely $d v=d t+\sqrt{g_{r r} / g_{t t}} d r$.

${ }^{18}$ We would like to thank Alex Buchel for a discussion on this point.
} 
for the perturbation: ${ }^{19}$

$$
S=\frac{1}{16 \pi G_{5}} \int \frac{d^{4} k}{(2 \pi)^{4}} d r \sum_{p, q=0}^{2} \mathcal{A}_{p, q}(r, k) \phi^{(p)}(r,-k) \phi^{(q)}(r, k) .
$$

Here, we use the Fourier transform to work in the momentum space $k=\{-\omega, \vec{k}\}$

$$
\Phi(r, x)=\int \frac{d^{4} k}{(2 \pi)^{4}} e^{i k \cdot x} \phi(r, k), \quad \phi(r,-k)=\phi^{*}(r, k)
$$

and $\phi^{(p)}(r, k)$ denotes the $p^{\text {th }}$ derivative of the field $\phi(r, k)$ with respect to $r(p+q \leq 2)$.

Next, we integrate by parts to obtain the bulk action for the graviton in the following form (up to some total derivative terms):

$$
S=\frac{1}{16 \pi G_{5}} \int \frac{d^{4} k}{(2 \pi)^{4}} d r\left[\mathcal{A}_{1}(r, k) \phi^{\prime}(r, k) \phi^{\prime}(r,-k)+\mathcal{A}_{0}(r, k) \phi(r, k) \phi(r,-k)\right],
$$

where

$$
\mathcal{A}_{1}(r, k)=-\frac{1}{2} e^{2 V(r)+W(r)} U(r), \quad \mathcal{A}_{0}(r, k)=\frac{e^{2 V(r)+W(r)} \omega^{2}}{2 U(r)} .
$$

At this point, it is important to emphasize that there are many total derivatives in this action that do not affect the equations of motion for the graviton. For the computation of the imaginary part of the two-point function, the coefficient of the term $\phi^{\prime} \phi^{\prime}$ in the bulk action is important. The other total derivatives in the bulk action and the Gibbons-Hawking surface term contribution exactly cancel on the boundary [27]. It was also shown [27] (it is straightforwardly to check it also in our case) that, in the case of Einstein gravity, the ratio of viscosity and entropy density is not affected when the matter fields are minimally coupled. The effective coupling $[27,71]$ is

$$
K_{\mathrm{eff}}=\frac{1}{16 \pi G_{5}} \frac{\mathcal{A}_{1}(r, k)}{\sqrt{-g} g^{r r}}=-\frac{1}{32 \pi G_{5}}
$$

and so the viscosity coefficient of the boundary fluid stress tensor is

$$
\eta=e^{2 V\left(r_{h}\right)+W\left(r_{h}\right)}\left(-2 K_{\mathrm{eff}}\left(r_{h}\right)\right) .
$$

In this case, the shear viscosity to entropy density ratio turns out to be universal, namely

$$
\frac{\eta}{s}=\frac{1}{4 \pi}
$$

\footnotetext{
${ }^{19}$ The terms that contain the derivatives with respect to the spatial coordinates, $\vec{x}$, combine in terms whose coefficient is proportional with $\vec{p}^{2}$. Since we work in the hydrodynamic approximation $\vec{p}=0$, these terms do not play any role in our analysis.
} 


\subsection{Four derivative action}

Let us now consider the action (2.1) supplemented with the most general four-derivative interactions [28]:

$$
\begin{aligned}
S_{\mathrm{HD}}=S_{E M}+\frac{\alpha^{\prime}}{16 \pi G_{5}} \int d^{5} x \sqrt{-g}[ & c_{1} R_{a b c d} R^{a b c d}+c_{2} R_{a b c d} F^{a b} F^{c d}+c_{3}\left(F^{2}\right)^{2} \\
& \left.+c_{4} F^{4}+c_{5} \epsilon^{a b c d e} A_{a} R_{b c f g} R_{d e}^{f g}\right]
\end{aligned}
$$

Since in supergravity actions the gauge kinetic terms couple to various scalars, it will be interesting to understand the role of the moduli in computing the viscosity bound. Unlike [28], our action contains a scalar, $\varphi$, and the coefficients $c_{i}$ depend on the value of $\varphi$. This resembles the four-derivative supergravity action $[72,73]$.

We treat the higher derivative terms perturbatively and apply the method of $[25,26]$ to compute the shear viscosity coefficient of the boundary fluid. However, to obtain the viscosity bound we also need the entropy density. We start by using the Noether charge formalism of Wald [74] (see, also, [48, 75] for a discussion in AdS) to compute the entropy density - we will need just the data in section 3.1 and appendix B.

When we add higher derivative corrections to the action, the entropy is no longer given by the area law — instead, we use a general formula proposed by Wald

$$
s=-2 \pi \int_{\mathcal{H}} \frac{\partial L}{\partial R_{a b c d}} \epsilon_{a b} \epsilon_{c d}
$$

where $\epsilon_{a b}$ is the binormal to the surface $\mathcal{H}$.

By using (4.10), we obtain the following expression for the entropy density:

$$
s=\frac{r_{h}^{3}}{4 G_{5}}+\frac{\alpha^{\prime}}{4 G_{5}} r_{h}^{3}\left[c_{1}\left(3 z_{1}^{2} r_{h}^{2}-4 u_{2}\right)-2 c_{2} q^{2}\right]+O\left(\alpha^{\prime 2}\right)
$$

We use the expression of $u_{2}$ given in appendix $\mathrm{B}$ to rewrite this expression as

$$
s=\frac{r_{h}^{3}}{4 G_{5}}-\frac{\alpha^{\prime} r_{h}^{3}}{G_{5}}\left[\frac{c_{1}}{3}\left(\frac{5 B^{2}}{r_{h}^{4}} e^{\alpha \varphi_{h}}+7 q^{2} e^{\alpha \varphi_{h}}-6\right)+\frac{c_{2}}{2} q^{2}\right]+O\left(\alpha^{\prime 2}\right) .
$$

As expected, the entropy density depends on four independent parameters, namely $\left(r_{h}, q, B, \varphi_{h}\right)$. Since in Wald formula only the four derivative interactions that involve the curvature tensor are important, the entropy only depends on $c_{1}$ and $c_{2}$ ( $c_{5}$ does not appear because the binormal has just $r t$ components and the contribution from this term vanishes).

To compute the four derivative corrections to the shear viscosity coefficient, we have to find the quadratic action for the transverse graviton moving in the background spacetime. As in previous section, we consider again the following metric perturbation

$$
g_{x y}=g_{x y}^{(0)}+h_{x y}(r, x)=g_{x y}^{(0)}[1+\epsilon \Phi(r, x)],
$$

where $\epsilon$ is an order counting parameter. 
In the presence of a generic $n$ derivative term in the bulk Lagrangian, the action (in momentum space) can be written as

$$
S=\frac{1}{16 \pi G_{5}} \int \frac{d^{4} k}{(2 \pi)^{4}} d r \sum_{p, q=0}^{n} \mathcal{A}_{p, q}(r, k) \phi^{(p)}(r,-k) \phi^{(q)}(r, k) .
$$

Here, $\phi^{(p)}(r, k)$ denotes the $p^{\text {th }}$ derivative of the field $\phi(r, k)$ with respect to $r$ and $p+q \leq n$. The coefficients $\mathcal{A}_{p, q}(r, k)$ depend, in general, on the coupling constant $\alpha^{\prime}$.

It is important to emphasize that $\mathcal{A}_{p, q}$ with $p+q \geq 3$ are proportional to $\alpha^{\prime}$ and vanish in $\alpha^{\prime} \rightarrow 0$ limit $[25,26]$ — these terms appear as an effect of higher derivative terms in the action.

This action does not have the canonical form as in the two derivative case. Therefore, it is not obvious how to generalize this approach for higher derivative case. The proof given in [24] was based on the canonical form of gravitons action. This problem was solved in $[25,26]$ and we use this method to compute the shear viscosity coefficient.

Let us now write the effective action for the transverse graviton in the canonical form with arbitrary coefficients:

$$
S_{\mathrm{eff}}=\frac{1}{16 \pi G_{5}} \int \frac{d \omega d^{3} \vec{k}}{(2 \pi)^{4}} d r\left[\mathcal{B}_{1}(r, k) \phi^{\prime}(r,-k) \phi^{\prime}(r, k)+\mathcal{B}_{0}(r, k) \phi(r, k) \phi(r,-k)\right] .
$$

We demand that the equations of motion obtained from the action (4.15) and original action (4.14) match at order $\alpha^{\prime}$. By comparing the equations of motion for $\phi(r, k)$ from the two actions, we get the function $\mathcal{B}_{0}$ and $\mathcal{B}_{1}$.

Once we have the effective action for $\phi(r, k)$ in the canonical form, the effective coupling can be easily read off:

$$
K_{\mathrm{eff}}(r)=\frac{1}{16 \pi G_{5}} \frac{\mathcal{B}_{1}(r, k)}{\sqrt{-g} g^{r r}}
$$

where $g^{r r}$ is the $r r$-component of the inverse perturbed metric and $\sqrt{-g}$ is the determinant of the perturbed metric. Therefore, the shear viscosity coefficient is

$$
\eta=r_{h}^{3}\left[-2 K_{e f f}\left(r=r_{h}\right)\right] .
$$

Evaluating the effective coupling in the near horizon we obtain the shear viscosity coefficient

$$
\begin{aligned}
\eta= & \frac{1}{16 \pi G_{5}} \\
& +\frac{\alpha^{\prime}\left(c_{1} r_{h}^{4}\left(8 q^{2} e^{\alpha \varphi_{h}}+3 z_{1}^{2} r_{h}^{2}-32 \pi T v_{1}-36 \pi T w_{1}-10 u_{2}+48\right)-B^{2}\left(c_{2}-8 c_{1} e^{\alpha \varphi_{h}}\right)\right)}{8 \pi G_{5} r_{h}^{4}} \\
& +\mathcal{O}\left(\alpha^{\prime 2}\right)
\end{aligned}
$$

which can be rewritten as (we use the results in appendix B)

$$
\eta=\frac{r_{h}^{3}}{16 \pi G_{5}}-\frac{\alpha^{\prime} r_{h}^{3}}{2 \pi G_{5}}\left[c_{1}\left(q^{2}+\frac{B^{2}}{r_{h}^{4}}\right) e^{\alpha \varphi_{h}}+\frac{c_{2}}{4} \frac{B^{2}}{r_{h}^{4}}\right]+\mathcal{O}\left(\alpha^{\prime 2}\right) .
$$


The ratio of the shear viscosity and entropy density turns out to be

$$
\frac{\eta}{s}=\frac{1}{4 \pi}+\frac{\alpha^{\prime}}{\pi}\left[\frac{c_{1}}{3}\left(\left(q^{2}-\frac{B^{2}}{r_{h}^{4}}\right) e^{\alpha \varphi_{h}}-6\right)+\frac{c_{2}}{2}\left(q^{2}-\frac{B^{2}}{r_{h}^{4}}\right)\right]+\mathcal{O}\left(\alpha^{\prime 2}\right) .
$$

In $B \rightarrow 0$ limit this result matches with $[28] .{ }^{20}$

Let us end up this section with a discussion of the extremal limit. In the absence of the moduli, the extremality condition is $2 B^{2}+r_{h}^{4}\left(q^{2}-6\right)=0$ and so the shear viscosity to entropy density ratio becomes

$$
\frac{\eta}{s}=\frac{1}{4 \pi}+\frac{\alpha^{\prime}}{\pi}\left[-c_{1} \frac{B^{2}}{r_{h}^{4}}+\frac{3 c_{2}}{2}\left(2-\frac{B^{2}}{r_{h}^{4}}\right)\right]+\mathcal{O}\left(\alpha^{\prime 2}\right) .
$$

Therefore, there is a drastic change when the magnetic field is turned on. That is, unlike the electrically charged solution studied in [28], the leading correction of $\eta / s$ in the extremal limit depends on both, $c_{1}$ and $c_{2}$. As expected, in $B \rightarrow 0$ limit our result matches with the one of [28].

In the presence of the scalar field, the extremal limit is more constrained. The scalar is fixed by the Chern-Simons coupling only, and by using some of the formulas derived in section 3, namely

$$
\begin{aligned}
2 B^{2} e^{\alpha \varphi_{h}}+r_{h}^{4}\left(q^{2} e^{\alpha \varphi_{h}}-6\right) & =0 \\
q^{2} r_{h}^{4}-B^{2} & =0 \\
\varphi_{h}-\frac{2}{3 \alpha} \ln |\zeta| & =0 .
\end{aligned}
$$

we obtain the following expression for the shear viscosity to entropy density ratio:

$$
\frac{\eta}{s}=\frac{1}{4 \pi}\left(1-8 c_{1} \alpha^{\prime}\right)+\mathcal{O}\left(\alpha^{\prime 2}\right) .
$$

Interestingly enough, we observe that there is no dependence of the horizon value of the modulus as in the non-extremal case. Also, the coefficient of $c_{2}$ vanishes and so the dependence of $c_{2}$ drops out.

\section{Discussion}

The goal of this paper was two-fold. First, to extend the work of $[15,16]$ by including scalar fields. Second, to compute the low frequency transport coefficients of a $(3+1)$-dimensional field theory whose gravity dual is an electrically and magnetically charged planar black hole in $\mathrm{AdS}_{5}$ Einstein-Maxwell theory with a gauge Chern-Simons term.

Since, in the extremal case, we have used the entropy function method to study the near horizon geometry, we were able to find analytic expressions for the entropy density and the near horizon parameters. In the absence of the scalar field we confirm the numerical results of [17], in particular the existence of a critical value of the magnetic field for which

\footnotetext{
${ }^{20}$ Note that our ' $q$ ' is different than ' $q$ ' of $[28]$. In [28], $q$ is the physical charge (up to some normalization). In our case, the physical charge is $\sim r_{h}^{6} q$.
} 
the entropy density vanishes. However, we have found a larger class of solutions - we have presented a detailed discussion in section 3 and we do not want to repeat the details here. We would also like to point out that one of the special values of the Chern-Simons coupling, $\zeta=1$, for which there exist finite horizon area solutions with both, electric and magnetic, fields non-zero corresponds to a special embedding of the gauge field $\mathrm{U}(1) \subset \mathrm{SU}(4)_{R}[15,16]$.

In [17], it was proposed that $(3+1)$-dimensional gauge theories holographically dual to $(4+1)$-dimensional Einstein-Maxwell-Chern-Simons theory undergo a quantum phase transition in the presence of a finite charge density and magnetic field. The authors of [17] also argue that a non-vanishing entropy density is 'exotic' from the point of view of CFTs that arise in the AdS/CFT duality. By turning on the magnetic field, instabilities can appear and this is consistent also with our analysis.

However, we believe that this result should be taken with caution. It is well known that, in many situations, by adding higher derivative corrections the singularities can be 'dressed' by horizons. ${ }^{21}$ Also, by turning on the moduli the geometries become more stable, though there can appear other kind of instabilities, e.g. the ones associated to the $A d S_{2}$ spacetime.

Since in string theory, typically, the gauge kinetic terms will couple to various scalars [20-23], we have considered a simple extension of $[15,16]$ by considering an exponential scalar coupling. In the presence of the moduli some important changes occur. First of all, in the non-extremal case, the near horizon data depend on the boundary value of the scalar. Therefore, the hydrodynamic properties of the dual field theories with higher derivative terms will be also controlled by the moduli.

Let us start with a discussion of the extremal case for which there is a drastic change. That is, due to the attractor mechanism, the near horizon geometry is universal and does not depend of the asymptotic values of the moduli. The horizon area is finite and so there is a large ground state degeneracy. One important question is what is happening in the presence of scalars? Is the ground state degeneracy still unstable under inclusion of a (large enough) magnetic field? Are there finite horizon area solutions for any value of the Chern-Simons coupling when both (magnetic and electric) fields are non-zero?

Before providing concrete answers to these questions, we would like to emphasize that the attractor mechanism plays an important role in classifying the bulk theories where the extremal entropy vanishes. A detailed discussion of the attractor mechanism in $\mathrm{AdS}_{5}$ and its embedding in string theory was presented in [61].

The universality of dual theory, which means that the IR physics does not depend upon the UV details, becomes in the holographic context the statement that the bulk solution near the horizon does not depend upon the details of the matter at large values of radial coordinate (boundary). Indeed, within the attractor mechanism, the black hole horizon (IR region) does not have any memory of the 'initial conditions' (UV values of the moduli) at the boundary. This is due to the existence of an infinite throat in the extremal near horizon geometry. In the presence of one gauge field (electric or magnetic) coupled with one scalar, the extremal limit is a naked (null) singularity. The reason is

\footnotetext{
${ }^{21}$ In [76], D'Hoker and Kraus have found a new solution with a near horizon geometry that does not contain an $A d S_{2}$. Based on the symmetries, one can argue that higher curvature corrections do not change the near horizon form of the solution.
} 
that the effective potential [54] does not have a minimum at the horizon. However, when the effective potential has a minimum at the horizon, there exist extremal solutions with finite horizon area. In our case we are not able to define an effective potential, but we have used, instead, the entropy function formalism. Therefore, the attractor mechanism can be regarded as a 'litmus test', which all extremal black holes with finite area should pass (the horizon values of the moduli should be finite).

In the presence of Chern-Simons term, there is no known concrete expression for the effective potential (there is a non-trivial fibration for $A d S_{2}$ ). Instead, we have used the entropy function and shown that the horizon value of the scalar is fixed. What came as a surprise was the fact that the modulus is fixed by the Chern-Simons coupling only. A simple argument ${ }^{22}$ of why is so is that, by rescaling the metric, we can connect the (near horizon extremal) solution in the absence of the modulus with the solution when the modulus is turned on. The starting point is a finite area solution and so we use the solution for $\zeta= \pm 1$ in the absence of the modulus. We then obtain a finite area extremal near horizon solution for which the modulus is fixed by the new Chern-Simons coupling. Therefore, in the presence of the moduli, there exist finite area horizon solutions for any value of the Chern-Simons coupling. We can also safely argue that the large ground state degeneracy is stable against scalar field perturbations. However, as in the case when the modulus is turned on, there also exists a critical value of the magnetic field and so we expect similar physical interpretations as the ones in [17].

Let us now comment on the second part of our paper. We have computed the shear viscosity to entropy density ratio in the presence of the most general bosonic four-derivative action (with one electromagnetic field) with moduli dependent couplings.

In general, there are two types of first-order corrections due to higher derivative terms. The entropy/area law is modified due to the additional terms in the action and/or the modification of the area due to the change of the metric at the horizon (the extra terms in the action change the equations of motion). Therefore, one has to use Wald formula to compute the entropy.

To compute the shear viscosity, we have used the method of $[25,26]$. Since we needed just the near horizon data, the computations are not very complicated even in the presence of higher derivative terms. We would like to emphasize that there is no ambiguity in defining the overall coefficient of the effective action and the results are consistent. Another important observation is that, in the presence of a magnetic field, there is a 'preferential' direction in the plasma. A priori it is not clear that the dual gravitational mode, $h_{x y}$, decouples. Indeed, that is not the case when the momentum is non-zero - however, in the hydrodynamic limit we have the required decoupling [70] and this is what we needed for the computation of the corresponding correlation function.

The shear viscosity to entropy density ratio is controlled by the horizon values of the scalars and so, in the non-extremal case, an operator deformation in the QFT will produce an interpolating non-trivial flow in which the moduli approach the (IR) black hole horizon. In the extremal case, though, the horizon moduli values are fixed and so the shear viscosity

\footnotetext{
${ }^{22}$ We would like to thank Rob Myers for a discussion on this point.
} 
to entropy density ratio does not depend on the asymptotic values of the scalars. Therefore, QFTs with different UV fixed points can flow to the same IR fixed point.

In the presence of the moduli, $\eta / s$ also depends only on the $c_{1}(\sim(c-a) / c$, where $c$ and $a$ are the central charges in the CFT) and $c_{2}$ (the coupling of the stress tensor to the $\mathrm{U}(1)$ current) coefficients. The other two coefficients, $c_{3}$ and $c_{4}$, parametrize couplings of the four-point function of the $\mathrm{U}(1)$ currents and so they should appear in the expressions of the charge density and conductivity [28].

It can be easily checked, that, when the scalars are turned off we recover the results of [28]. Since [28] contains a very detailed discussion on the relation with the previous results in the literature, we do not want to repeat it here. We would like to point out just that, in the extremal limit and in the presence of the magnetic field, we also obtain a deconfined 'plasma' in the dual CFT. However, in the presence of the magnetic field, $\eta / s$ depends on both coefficients, $c_{1}$ and $c_{2}$ - when the magnetic field vanishes, $\eta / s$ only depends on $c_{2}$ and we recover the result of [28]. In $N=2$ supergravity, the bulk supersymmetry constraints all four derivative couplings to be proportional to a single overall constant.

When the modulus is turned on, the extremal limit is more intriguing. The dependence of $\eta / s$ on $c_{2}$ drops out and so $\eta / s$ depends only on the central charges in the dual CFT. This is somehow similar with the $N=2$ supergravity case mentioned above for which all the coefficients are fixed by the central charges.

We close with some future directions.

Due to the presence of a Chern-Simons term, there is a more interesting kind of instability, ${ }^{23}$ which can appear $[77,78]$. This instability is caused by a non-normalizable mode of the $\mathrm{AdS}_{2}$ factor in the near horizon geometry. ${ }^{24}$ This instability appears for large enough Chern-Simons couplings and a finite momentum.

It will be interesting to check [79] if this kind of instability appears also in our case. We would like to point out that the near horizon geometry, when there are background electric and magnetic fields, is in fact a fibered $A d S_{2} \times R^{3}$, not a direct product as in [77, 78]. In this case an analysis of the near horizon geometry as in our paper (see, also, [80]) is more appropriate.

Using arguments as in [81], a computation of other transport coefficients is also possible [19]. However, since the near horizon data are not enough, a more involved analysis is needed. Another reason of why the analysis is more complicated is that the hydrodynamic limit does not commute with the limit of small magnetic fields. Therefore, one expects drastic changes of the transport properties of magnetized fluids. In this context, it will be interesting to study how the attenuation and the speed of sound waves are affected by the background magnetic field - a similar analysis for a $(2+1)$-dimensional plasma was presented in [70]. It will also be interesting to see if new dissipative transport coefficients can appear in the effective hydrodynamic description of plasmas in external magnetic fields [82].

\footnotetext{
${ }^{23}$ We would like to thank Hirosi Ooguri for pointing out this to us.

${ }^{24}$ It was shown in $[77,78]$ that the near horizon analysis gives a sufficient but not necessary condition. The reason is that, for the Reissner-Nordström case studied in [77, 78], there are unstable modes in the full bulk geometry that do not reduce to normalizable modes in the near horizon limit.
} 


\section{Acknowledgments}

We would like to thank Stefan Theisen for fruitful discussions and collaboration in the initial stage of this project. We would also like to thank Alex Buchel, Dileep Jatkar, Per Kraus, Prem Kumar, Rob Myers, Hirosi Ooguri, Bernard de'Wit for interesting discussions, and especially Ashoke Sen for valuable discussions and comments on an earlier draft of this paper. DA would like to thank ESI, Vienna for the hospitality during the last stages of this work and the organizers of ESI Programme on AdS Holography and the QuarkGluon Plasma for a stimulating environment. NB and SD acknowledge the hospitality of AEI, Potsdam at various stages of this work. NB is also thankful to Swansea University for the hospitality at the final stage of this work. The work of NB is a part of research programme of FOM, which is financially supported by the Netherlands Organization for Scientific Research (NWO).

\section{A Kubo formula and shear viscosity}

In this appendix we present a brief review of Kubo formula in the context of relativistic hydrodynamics. Since we are interested in computing the transport coefficients by using the AdS/CFT duality, we follow closely the nice reviews [5] (see, also, [83] for a good introduction in relativistic hydrodynamics).

Current understanding is that the matter produced in the heavy-ion experiments at RHIC behaves collectively like a fluid. That is, the system rapidly thermalizes and comes into local thermal equilibrium; then it evolves according to hydrodynamics until it hadronizes and the temperature becomes smaller than the deconfinement temperature.

Therefore, the relativistic hydrodynamics - in short, the Navier-Stokes equations and their relativistic generalizations - is the best method currently available for modeling the spacetime evolution of sQGP.

Hydrodynamics can be understood as an effective theory, which describes the dynamics at large length and time-scales. It relies only on the assumption of local equilibrium. ${ }^{25}$

In order to describe essential non-equilibrium phenomena in heavy-ion collision, a transport theory approach seems necessary. The first important step to investigate small perturbations of a high temperature hadronic matter is the linear response formalism.

If $l_{\mathrm{mfp}}$ is the mean free path - the average distance traversed between collisions by particles (of the liquid) - then, a perturbation of the liquid (in equilibrium) will disappear at distances of order $l_{\mathrm{mfp}}$. However, the fluctuations associated to some conservation laws can propagate without being damped off. For example, a perturbation of (mean) energy can propagate to infinity if there is no internal friction (viscosity) of the fluid.

In the rest of this section we would like to explain why a measure of $\eta / s$ is important and we also briefly review the Kubo's formula, which is useful for computing the viscosity.

Unlike in standard thermodynamics where the system is in global thermodynamic equilibrium (the intensive parameters, e.g. the pressure $(P)$ and temperature $(T)$, are

\footnotetext{
${ }^{25}$ One does not have to make other assumptions on the classical/quantum nature of the phenomena involved or the type of particles/fields and their interactions.
} 
constant throughout the volume) and at rest, we are interested in systems whose pressure and temperature vary with space and time, and which are not at rest.

Though, we request that the system is in local thermodynamic equilibrium. That is, the fluid is characterized only by local temperature and velocity fields that vary slowly on the scale set by the temperature. Consequently, one can assume the thermodynamic equilibrium in some neighborhood about any point.

In hydrodynamics, it is useful to work with densities per unit volume: the energy density $\epsilon \equiv U / V$ and the entropy density $s \equiv S / V$. The usual relation for the energy can be rewritten as $T s=\epsilon+P$ - note that all these densities are intensive quantities. ${ }^{26}$

In hydrodynamics, we work directly with the equations of motion and assume an expansion in derivatives so that $\partial_{\mu} \ll l_{\mathrm{mfp}}^{-1}$. We express the stress tensor through the temperature $T(x)$ and velocity $u^{\mu}(x)$.

For an ideal fluid, the hydrodynamic equations are equivalent with the laws of conservation of energy and momentum:

$$
\partial_{\mu} T^{\mu \nu}=0, \quad T^{\mu \nu}=(\epsilon+P) u^{\mu} u^{\nu}+P g^{\mu \nu} .
$$

It is clear from the above expression that the momentum density is $(\epsilon+P) \vec{v}$ and so, unlike the non-relativistic fluid, the pressure contributes to the inertia of a relativistic fluid.

At the next order we obtain the energy momentum tensor of a relativistic viscous fluid whose conservation equation reproduces the relativistic version of the Navier-Stokes equation. The stress tensor at this order becomes

$$
T^{\mu \nu}=P g^{\mu \nu}+(\epsilon+P) u^{\mu} u^{\nu}-\sigma^{\mu \nu}
$$

with the dissipative part

$$
\sigma^{\mu \nu}=P^{\mu \alpha} P^{\nu \beta}\left[\eta\left(\partial_{\alpha} u_{\beta}+\partial_{\beta} u_{\alpha}-\frac{2}{3} g_{\alpha \beta} \partial_{\mu} u^{\mu}\right)+\varrho g_{\alpha \beta} \partial_{\mu} u^{\mu}\right] .
$$

Here, $P^{\mu \nu}=g^{\mu \nu}+u^{\mu} u^{\nu}$ is the usual projector operator: for a fluid at rest it becomes $P^{\mu \nu}=\operatorname{diag}(0,+1,+1,+1)$ and so it projects on space.

The numerical coefficient of the traceless part, $\eta$, is called the shear viscosity. The numerical coefficient of the trace part, $\varrho$, is called bulk viscosity.

To understand better the physical interpretation of the transport coefficients and why a measure of $\eta / s$ (and $\varrho / s$ ) is relevant (see, also, [84]), let us work in the fluid rest frame $u^{\mu}=(1,0,0,0)$ and consider a small fluctuation around the thermal equilibrium: $u^{i} \ll 1$, $u^{0} \simeq 1+O\left(u^{i} u^{i}\right), T=T+\delta T$, etcetera.

For the ideal fluid, we find the following perturbed stress tensor at first order:

$$
T^{00}=\epsilon+\delta \epsilon, \quad T^{0 i}=(\epsilon+P) u^{i}, \quad T^{i j}=(P+\delta P) \delta^{i j} .
$$

\footnotetext{
${ }^{26}$ All thermodynamic quantities associated with a fluid element, e.g. $s, \epsilon$, and $P$, are defined in the rest frame and so they are Lorentz scalars by construction.
} 
The deviation from the ideal fluid stress tensor (at first order) in the fluid rest frame (we use the properties of the projector) is

$$
\begin{aligned}
\delta T_{i j} & =\sigma_{i j}=\eta\left(\partial_{j} u_{i}+\partial_{i} u_{j}-\frac{2}{3} \delta_{i j} \partial_{k} u^{k}\right)+\varrho \delta_{i j} \partial_{k} u^{k} \\
& =\frac{\eta}{T s}\left(\partial_{j} T_{i}^{0}+\partial_{i} T_{j}^{0}-\frac{2}{3} \delta_{i j} \partial_{k} T^{k 0}\right)+\frac{\varrho}{T s} \delta_{i j} \partial_{k} T^{k 0} .
\end{aligned}
$$

In the last expression we used (A.3) and $T s=\epsilon+P$.

Now it is clear that, since the temperature is the only relevant energy scale (experimental parameter), the viscous terms are characterized by the coefficients $\eta / s$ and $\varrho / s$ (medium parameters). The physical interpretation is as follows: the bulk viscosity encodes the resistance of the system to uniform expansion and the shear viscosity controls the rate of the momentum diffusion in the transverse direction to the flow.

For conformal fluids the energy-momentum tensor should be traceless in flat space, which implies $\varrho=0$ and $\epsilon=3 P$.

We have defined the dissipative coefficients as phenomenological constants. However, it is important to find a way to compute them directly from the microscopic theory. The computation of the shear viscosity in quantum field theory relies on the Kubo formula the dual models of CFTs are well suited to evaluate Kubo expressions.

Let us now briefly present Kubo formula and its physical interpretation.

We are interested to understand the response of a macroscopic quantum (many-body) system to a localized disturbance, which is created by an applied external force. Let us consider a local observable $O(\vec{x}, t)$ (e.g., the charge current or $T^{\mu \nu}$, which is a set of conserved currents) and an external source that couples linearly to the observable so that the new action is

$$
S=S_{0}+\int d x O(x) J(x)
$$

The response, which is the change (from zero at the equilibrium) in the expectation value of the observable induced by the source, is linear in the source:

$$
<O(x)>=\int d y \chi(x-y) J(y) \equiv \chi \cdot J .
$$

The coefficient of proportionality is called 'susceptibility' and is nothing else than the retarded Green function of the physical observable:

$$
\chi(x-y) \equiv-\frac{i}{\hbar} \theta\left(x^{0}-y^{0}\right)<[O(x), O(y)]>.
$$

One can also Fourier transform to obtain that, indeed,

$$
\chi(\omega, \vec{p})=\frac{<O(\omega, \vec{p})>}{J(\omega, \vec{p})} .
$$

Let us know find the Kubo formula for viscosity (see, e.g., [5]). We consider a perturbation of the metric so that the only non-zero component $h_{12}(t)$ does not depend of space 
coordinates (it corresponds to $\vec{p}=0$ in momentum space). Obviously, this perturbation can not excite the temperature (which is a scalar) and the velocity (which is a vector). One can easily compute the 12-component of the stress tensor (by using the covariant derivative) to obtain

$$
\eta=-\lim _{\omega \rightarrow 0} \frac{1}{\omega} \operatorname{Im}\left(\int d^{4} x \theta(t) e^{i \omega t}<\left[T_{12}(x), T_{12}(0)\right]>\right) .
$$

\section{B Coefficients in expansion (3.3) at $\mathcal{O}\left(r-r_{h}\right)^{2}$}

For completeness, in this appendix we present the other coefficients that appear in the near horizon expansion of different fields in eq. (3.3). They are

$$
\begin{aligned}
u_{2}= & e^{\alpha \varphi_{h}}\left(\frac{5 B^{2}}{3 r_{h}^{4}}+\frac{7 q^{2}}{3}\right)+\frac{3}{4} z_{1}^{2} r_{h}^{2}-2 \\
v_{2}= & \frac{1}{288 \pi^{2} T^{2}}\left(e ^ { - 2 \alpha \phi _ { h } } \left(24 B^{4} \zeta^{2} e^{\alpha \phi_{h}}+24\left(5 B^{2}+2 q^{2}\right) e^{3 \alpha \phi_{h}}-72 B^{2} \zeta^{2}\right.\right. \\
& \left.\left.+\left(6 B^{4}\left(\alpha^{2}-4\right)-B^{2} q^{2}\left(9 \alpha^{2}+8\right)+q^{4}\left(3 \alpha^{2}-4\right)\right) e^{4 \alpha \phi_{h}}-144 e^{2 \alpha \phi_{h}}\right)\right) \\
w_{2}= & \frac{e^{-4 \alpha \phi_{h}}}{288 \pi^{2} T^{2}}\left(-36 B^{4} \zeta^{4}-24 B^{2} \zeta^{2}\left(B^{2}+q^{2}\right) e^{3 \alpha \phi_{h}}+48\left(q^{2}-2 B^{2}\right) e^{5 \alpha \phi_{h}}+288 B^{2} \zeta^{2} e^{2 \alpha \phi_{h}}\right. \\
& \left.+\left(-3 B^{4}\left(\alpha^{2}-4\right)-8 B^{2} q^{2}+q^{4}\left(3 \alpha^{2}-4\right)\right) e^{6 \alpha \phi_{h}}-144 e^{4 \alpha \phi_{h}}\right) \\
z_{2}= & -\frac{B \zeta\left(B^{2}\left(9 \zeta^{2} e^{-3 \alpha \phi_{h}}+1\right)+5\left(q^{2}-6 e^{-\alpha \phi_{h}}\right)\right)}{6 \pi T} \\
p_{1}= & \frac{B q \zeta e^{-3 \alpha \phi_{h}}\left(\left(3 \alpha^{2}+4\right)\left(B^{2}-q^{2}\right) e^{3 \alpha \phi_{h}}-12 B^{2} \zeta^{2}+24 e^{2 \alpha \phi_{h}}\right)}{96 \pi^{2} T^{2}} .
\end{aligned}
$$

\section{Details on entropy function when the modulus is turned on}

In the presence of the modulus, the attractor equation are:

$$
\begin{aligned}
-\frac{\beta \pi \sqrt{v_{2}}}{L v_{1}^{2}}\left[4 B^{2} L^{2} e^{\alpha \varphi_{h}}+v_{1}^{2}\left(-4 L+24 L^{2}+4 q^{2} e^{\alpha \varphi_{h}}+v_{2} z 1^{2}\right)\right] & =0 \\
\frac{\beta \pi}{L v_{1} \sqrt{v_{2}}}\left[4 B^{2} L^{2} e^{\alpha \varphi_{h}}-v_{1}^{2}\left(-4 L+24 L^{2}+4 q^{2} e^{\alpha \varphi_{h}}+3 v_{2} z_{1}^{2}\right)\right] & =0 \\
\frac{\beta \pi \sqrt{v_{2}}}{L v_{1}^{2}}\left[4 B^{2} L^{2} e^{\alpha \varphi_{h}}+v_{1}^{2}\left(-24 L^{2}+4 q^{2} e^{\alpha \varphi_{h}}+v_{2} z_{1}^{2}\right)\right] & =0 \\
\frac{2 \beta \pi}{L}\left[L\left(\Theta-4 B p_{1}^{2} \zeta\right)+v_{1} \sqrt{v_{2}}\left(4 p_{1} q e^{\alpha \varphi_{h}}-v_{2} z 1\right)\right] & =0 \\
\frac{8 \beta \pi q}{L}\left[2 B L \zeta+v_{1} \sqrt{v_{2}} z_{1} e^{\alpha \varphi_{h}}\right] & =0 \\
2 \pi \beta\left(8 B p_{1} \zeta-\frac{4 v_{1} \sqrt{v_{2}} q e^{\alpha \varphi_{h}}}{L}+Q\right) & =0 \\
-2 \beta e^{\alpha \varphi_{h}} L v_{1} \sqrt{v_{2}} \pi\left(-\frac{2 B^{2}}{v_{1}^{2}}+\frac{2 q^{2}}{L^{2}}\right) \alpha & =0
\end{aligned}
$$


From the last equation, we observe that, in the presence of the modulus, a non-trivial extremal black hole can be obtained if neither $q$ nor $B$ vanish. Following the same steps as in section 3.2.2, we can obtain the near horizon geometry. First we compute the following quantities:

$$
\begin{aligned}
q & =\frac{e^{-\alpha \varphi_{h}} L\left(Q+8 B p_{1} \zeta\right)}{4 v_{1} \sqrt{v_{2}}} \\
z_{1} & =\frac{L\left(p_{1} Q+\Theta+4 B p_{1}^{2} \zeta\right)}{v_{1} v_{2}^{\frac{3}{2}}} \\
p_{1} & =\frac{-Q+\sqrt{Q^{2} \pm 16 B\left(2 B E^{\frac{\alpha \varphi_{h}}{2}} v_{2} \mp \Theta\right) \zeta}}{8 B \zeta} \\
v_{2} & =\frac{Q^{2}-16 B \Theta \zeta}{32 e^{\alpha \varphi_{h}}\left(3 v_{1}^{2}-B^{2} e^{-\frac{\alpha \varphi_{h}}{2}}\left(e^{\frac{3 \alpha \varphi_{h}}{2}} \pm \zeta\right)\right)} .
\end{aligned}
$$

With these results, the constraint (5th) relation becomes

$$
\frac{B}{36 v_{1}} e^{-\alpha \varphi_{h} / 2}\left(e^{\frac{3 \alpha \varphi_{h}}{2}} \pm \zeta\right) \sqrt{\frac{3 v_{1}^{2}-B^{2} e^{\alpha \varphi_{h}}}{2}}=0
$$

and we obtain the following expression for the entropy:

$$
S=\beta v_{1} \pi \sqrt{\frac{Q^{2}-16 B \Theta \zeta}{2 e^{\frac{\alpha \varphi_{h}}{2}}\left(3 v_{1}^{2} e^{\frac{\alpha \varphi_{h}}{2}}-B^{2}\left(e^{\frac{3 \alpha \varphi_{h}}{2}} \pm \zeta\right)\right)}} .
$$

Now, let us rewrite the near horizon geometry in terms of the charges

$$
\begin{aligned}
q & =\frac{1}{6 v_{1}} e^{-\alpha \varphi_{h} / 2} \sqrt{\frac{3 v_{1}^{2}-B^{2} e^{\alpha \varphi_{h}}}{2}} \\
z_{1} & = \pm \frac{2 B e^{\frac{\alpha \varphi_{h}}{2}}}{3 v_{1} \sqrt{\frac{Q^{2}-16 B \Theta \zeta}{2 e^{\frac{\alpha \varphi_{h}}{2}}\left(3 v_{1}^{2} e^{\frac{\alpha \varphi_{h}}{2}}-B^{2}\left(e^{\frac{3 \alpha \varphi_{h}}{2}} \pm \zeta\right)\right)}}} .
\end{aligned}
$$

By using the last equation of C.1 we obtain

$$
v_{1}= \pm \frac{B e^{\alpha \varphi_{h} / 2}}{\sqrt{2}}
$$

and from the fifth relation of C.1, we see that the only possibility is a fixed horizon value of the scalar:

$$
\varphi_{h}=\frac{2}{3} \ln |\zeta|
$$

What comes as a surprise is the fact that the horizon value of the scalar is fixed by the Chern-Simons coupling. Consequently, the entropy is

$$
S=\beta \pi \sqrt{\frac{Q^{2}-16 B \Theta \zeta}{6|\zeta|^{2 / 3}}} .
$$




\section{Decoupling of $h_{x y}$ mode}

In what follows, we provide a detailed derivation of the decoupling of the dual gravitational mode (4.1). We have explicitly checked that the $h_{x y}=e^{i t \omega+2 V(r)} \epsilon \Phi(r)$ mode does not couple with any other field when the momentum vanishes.

For two derivative gravity theory, this can be easily seen from the equations of motion (2.3), (2.4), (2.5). However, we are interested in the most general four-derivative action (4.9). In this case, instead of writing the equations of motion in the presence of higher derivative terms, we will explicitly compute the action up to order $\epsilon^{2}$. In this way, it can be explicitly checked that there is no coupling between $h_{x y}$ and the other fields.

Let us turn on the following perturbations of the metric

$$
\begin{aligned}
& g_{\alpha \beta}=g_{\alpha \beta}^{(0)}+\epsilon h_{\alpha \beta} \\
& =\left(\begin{array}{ccccc}
\frac{1}{U(r)}+e^{i t \omega} \epsilon \xi_{1}(r) & e^{i t \omega} \epsilon \xi_{2}(r) & e^{i t \omega} \epsilon \xi_{3}(r) & e^{i t \omega} \epsilon \xi_{4}(r) & e^{i t \omega} \epsilon \xi_{5}(r) \\
e^{i t \omega} \epsilon \xi_{2}(r) & e^{2 W(r)} Z(r)^{2}-U(r) & e^{i t \omega} \epsilon \Upsilon(r) & 0 & e^{2 W(r)} Z(r)+e^{i t \omega} \epsilon v(r) \\
e^{i t \omega} \epsilon \xi_{3}(r) & e^{i t \omega} \epsilon \Upsilon(r) & e^{2 V(r)} & e^{i t \omega+2 V(r)} \epsilon \Phi(r) & e^{i t \omega} \epsilon \chi(r) \\
e^{i t \omega} \epsilon \xi_{4}(r) & 0 & e^{i t \omega+2 V(r)} \epsilon \Phi(r) & e^{2 V(r)} & 0 \\
e^{i t \omega} \epsilon \xi_{5}(r) & e^{2 W(r)} Z(r)+e^{i t \omega} \epsilon v(r) & e^{i t \omega} \epsilon \chi(r) & 0 & e^{2 W(r)}
\end{array}\right)
\end{aligned}
$$

and gauge gauge fields

$$
\begin{aligned}
A_{\alpha} & =A_{\alpha}^{(0)}+\epsilon f_{\alpha} \\
& =\left(e^{i t \omega} \epsilon a_{r}(r), e^{i t \omega} \epsilon a_{t}(r)-E(r), \frac{B y}{2}+e^{i t \omega} \epsilon a_{x}(r),-\frac{B x}{2}+e^{i t \omega} \epsilon a_{y}(r), e^{i t \omega} \epsilon a_{z}(r)+P(r)\right)
\end{aligned}
$$

Here $g_{\alpha \beta}^{(0)}$ and $A_{\alpha}^{(0)}$ are the background metric (2.8) and the background gauge field; $\epsilon$ dependent terms are the perturbations.

Using these field excitations, we can now compute the action. ${ }^{27}$ The result is complicated, but for our purpose it is enough to pick up the $\Phi(r)$-dependent terms — for

\footnotetext{
${ }^{27}$ We use the Mathematica notebook for this computation. We emphasize that our system is symmetric in $x$ - and $y$-directions.
} 
concretness, let us write the $\Phi(r)$-dependent part of the action:

$$
\begin{aligned}
S_{\Phi, \Phi}= & \Phi(r)^{2}\left[\frac { 1 } { 4 U ( r ) } \left(\left(e ^ { - 2 V ( r ) - W ( r ) } \left(-U(r) e^{2 W(r)}\left(4 B^{2} e^{\alpha \varphi(r)}+4 Q(r)^{2} e^{4 V(r)+\alpha \varphi(r)}\right.\right.\right.\right.\right. \\
& \left.-2 e^{4 V(r)} U^{\prime \prime}(r)-4 e^{4 V(r)} U^{\prime}(r)\left(2 V^{\prime}(r)+W^{\prime}(r)\right)+e^{4 V(r)+2 W(r)} Z^{\prime}(r)^{2}+24 e^{4 V(r)}\right) \\
& +2 U(r)^{2} e^{4 V(r)}\left(2 P^{\prime}(r)^{2} e^{\alpha \varphi(r)}+e^{2 W(r)}\left(4 V^{\prime \prime}(r)+4 V^{\prime}(r) W^{\prime}(r)+6 V^{\prime}(r)^{2}\right.\right. \\
& \left.\left.\left.\left.\left.\left.+2 W^{\prime \prime}(r)+2 W^{\prime}(r)^{2}-\varphi^{\prime}(r)^{2}\right)\right)+14 \omega^{2} e^{4 V(r)+2 W(r)}\right)\right)\right]\right] \\
& +\Phi(r) \Phi^{\prime}(r)\left[\left(2 e^{2 V(r)+W(r)}\left(U^{\prime}(r)+U(r)\left(3 V^{\prime}(r)+W^{\prime}(r)\right)\right)\right.\right. \\
& -\left(2 \alpha ^ { \prime } e ^ { W ( r ) - 2 V ( r ) } \left(-2 U(r) V^{\prime}(r)\left(U(r)\left(B^{2} c_{2}+c_{1} e^{4 V(r)+2 W(r)} Z^{\prime}(r)^{2}\right)-2 c_{1} U(r)^{2} e^{4 V(r)}\right.\right.\right. \\
& \left.\left.+2 V^{\prime \prime}(r)+3 V^{\prime}(r)^{2}+W^{\prime}(r)^{2}\right)+2 c_{1} \omega^{2} e^{4 V(r)}\right)+2 c_{1} U(r) e^{4 V(r)} U^{\prime}(r)^{2} V^{\prime}(r) \\
& \left.\left.\left.\left.+c_{1} e^{4 V(r)} U^{\prime}(r)\left(2 U(r)^{2}\left(V^{\prime \prime}(r)+3 V^{\prime}(r)^{2}\right)+\omega^{2}\right)\right)\right) / U(r)\right)\right] \\
& +\Phi(r) \Phi^{\prime \prime}(r)\left[\left(2 U(r) e^{2 V(r)+W(r)}-4 c_{1} \alpha^{\prime} U(r) e^{2 V(r)+W(r)}\left(U^{\prime}(r) V^{\prime}(r)\right.\right.\right. \\
& \left.\left.\left.+2 U(r)\left(V^{\prime \prime}(r)+V^{\prime}(r)^{2}\right)\right)\right)\right] \\
& +\Phi^{\prime}(r)^{2}\left[\left(\alpha ^ { \prime } e ^ { W ( r ) - 2 V ( r ) } \left(U(r)\left(B^{2} c_{2}+2 c_{1} e^{4 V(r)} U^{\prime}(r) V^{\prime}(r)-c_{1} e^{4 V(r)+2 W(r)} Z^{\prime}(r)^{2}\right)\right.\right.\right. \\
& \left.+c_{1} e^{4 V(r)}\left(U^{\prime}(r)^{2}+4 \omega^{2}\right)+2 c_{1} U(r)^{2} e^{4 V(r)}\left(-2 V^{\prime \prime}(r)+V^{\prime}(r)^{2}+W^{\prime}(r)^{2}\right)\right) \\
& \left.\left.+\frac{3}{2} U(r) e^{2 V(r)+W(r)}\right)\right] \\
& +\Phi^{\prime}(r) \Phi^{\prime \prime}(r)\left[\left(2 c_{1} \alpha^{\prime} U(r) e^{2 V(r)+W(r)}\left(U^{\prime}(r)+4 U(r) V^{\prime}(r)\right)\right)\right] \\
& +\Phi^{\prime \prime}(r)^{2}\left[2 c_{1} \alpha^{\prime} U(r)^{2} e^{2 V(r)+W(r)]}\right.
\end{aligned}
$$

Since there is no mixing between $\Phi$ and the other modes in the zero momentum limit, this mode remains massless and a computation of the related Green's function from the near-horizon data is still possible.

However, the other metric fluctuations namely $h_{x z}$ or $h_{y z}$ are not decoupled from the gauge field perturbations in the zero momentum limit (even in the absence of higher derivative terms) - these coupled terms are proportionl to B or $\zeta$ (CS term). Therefore, these modes are not massless in the zero momentum limit and the near horizon geometry is not sufficient to compute the related two point correlation functions, i.e. $\left\langle\left[T_{x z}, T_{x z}\right]>\right.$ or $<\left[T_{y z}, T_{y z}\right]>$.

Open Access. This article is distributed under the terms of the Creative Commons Attribution Noncommercial License which permits any noncommercial use, distribution, and reproduction in any medium, provided the original author(s) and source are credited. 


\section{References}

[1] K. Rajagopal and F. Wilczek, The condensed matter physics of QCD, hep-ph/0011333 [SPIRES].

[2] P.F. Kolb and U.W. Heinz, Hydrodynamic description of ultrarelativistic heavy-ion collisions, nucl-th/0305084 [SPIRES].

[3] J.M. Maldacena, The large-N limit of superconformal field theories and supergravity, Int. J. Theor. Phys. 38 (1999) 1113 [Adv. Theor. Math. Phys. 2 (1998) 231] [hep-th/9711200] [SPIRES].

[4] V.I. Zakharov, Old problems of QCD phenomenology in view of AdS/QCD correspondence, AIP Conf. Proc. 964 (2007) 143 [SPIRES].

[5] D.T. Son, Applied holography, Nucl. Phys. Proc. Suppl. 195 (2009) 217 [SPIRES].

[6] S. Bhattacharyya, V.E. Hubeny, S. Minwalla and M. Rangamani, Nonlinear Fluid Dynamics from Gravity, JHEP 02 (2008) 045 [arXiv:0712.2456] [SPIRES].

[7] G. Policastro, D.T. Son and A.O. Starinets, The shear viscosity of strongly coupled $N=4$ supersymmetric Yang-Mills plasma, Phys. Rev. Lett. 87 (2001) 081601 [hep-th/0104066] [SPIRES].

[8] P. Kovtun, D.T. Son and A.O. Starinets, Viscosity in strongly interacting quantum field theories from black hole physics, Phys. Rev. Lett. 94 (2005) 111601 [hep-th/0405231] [SPIRES].

[9] A. Sinha and R.C. Myers, The viscosity bound in string theory, Nucl. Phys. A 830 (2009) 295c-298c [arXiv:0907.4798] [SPIRES].

[10] K. Fukushima, M. Ruggieri and R. Gatto, Chiral magnetic effect in the PNJL model, Phys. Rev. D 81 (2010) 114031 [arXiv: 1003.0047] [SPIRES].

[11] W.-j. Fu, Y.-x. Liu and Y.-l. Wu, Chiral Magnetic Effect and QCD Phase Transitions with Effective Models, arXiv:1003.4169 [SPIRES].

[12] K. Fukushima, D.E. Kharzeev and H.J. Warringa, Electric-current Susceptibility and the Chiral Magnetic Effect, Nucl. Phys. A 836 (2010) 311 [arXiv:0912.2961] [SPIRES].

[13] D.E. Kharzeev and H.J. Warringa, Chiral Magnetic conductivity, Phys. Rev. D 80 (2009) 034028 [arXiv:0907.5007] [SPIRES].

[14] K. Fukushima, D.E. Kharzeev and H.J. Warringa, The Chiral Magnetic Effect, Phys. Rev. D 78 (2008) 074033 [arXiv:0808.3382] [SPIRES].

[15] E. D'Hoker and P. Kraus, Charged Magnetic Brane Solutions in AdS $S_{5}$ and the fate of the third law of thermodynamics, JHEP 03 (2010) 095 [arXiv:0911.4518] [SPIRES].

[16] E. D'Hoker and P. Kraus, Magnetic Brane Solutions in AdS, JHEP 10 (2009) 088 [arXiv:0908.3875] [SPIRES].

[17] E. D'Hoker and P. Kraus, Holographic Metamagnetism, Quantum Criticality and Crossover Behavior, JHEP 05 (2010) 083 [arXiv:1003.1302] [SPIRES].

[18] D. Astefanesei, N. Banerjee and S. Dutta, Near horizon analysis of extremal AdS black holes, to appear.

[19] D. Astefanesei, N. Banerjee and S. Dutta, Conductivity of electromagnetic black branes, work in progress. 
[20] J. Maldacena, D. Martelli and Y. Tachikawa, Comments on string theory backgrounds with non-relativistic conformal symmetry, JHEP 10 (2008) 072 [arXiv:0807.1100] [SPIRES].

[21] D. Cassani, G. Dall'Agata and A.F. Faedo, Type IIB supergravity on squashed Sasaki-Einstein manifolds, JHEP 05 (2010) 094 [arXiv: 1003.4283] [SPIRES].

[22] J.T. Liu, P. Szepietowski and Z. Zhao, Consistent massive truncations of IIB supergravity on Sasaki-Einstein manifolds, Phys. Rev. D 81 (2010) 124028 [arXiv:1003.5374] [SPIRES].

[23] J.P. Gauntlett and O. Varela, Universal Kaluza-Klein reductions of type IIB to $N=4$ supergravity in five dimensions, JHEP 06 (2010) 081 [arXiv: 1003.5642] [SPIRES].

[24] N. Iqbal and H. Liu, Universality of the hydrodynamic limit in AdS/CFT and the membrane paradigm, Phys. Rev. D 79 (2009) 025023 [arXiv:0809.3808] [SPIRES].

[25] N. Banerjee and S. Dutta, Higher Derivative Corrections to Shear Viscosity from Graviton's Effective Coupling, JHEP 03 (2009) 116 [arXiv:0901.3848] [SPIRES].

[26] N. Banerjee and S. Dutta, Shear Viscosity to Entropy Density Ratio in Six Derivative Gravity, JHEP 07 (2009) 024 [arXiv:0903.3925] [SPIRES].

[27] R.-G. Cai, Z.-Y. Nie and Y.-W. Sun, Shear Viscosity from Effective Couplings of Gravitons, Phys. Rev. D 78 (2008) 126007 [arXiv:0811.1665] [SPIRES].

[28] R.C. Myers, M.F. Paulos and A. Sinha, Holographic Hydrodynamics with a Chemical Potential, JHEP 06 (2009) 006 [arXiv:0903.2834] [SPIRES].

[29] R.-G. Cai, Z.-Y. Nie, N. Ohta and Y.-W. Sun, Shear Viscosity from Gauss-Bonnet Gravity with a Dilaton Coupling, Phys. Rev. D 79 (2009) 066004 [arXiv:0901.1421] [SPIRES].

[30] X.-H. Ge and S.-J. Sin, Shear viscosity, instability and the upper bound of the Gauss-Bonnet coupling constant, JHEP 05 (2009) 051 [arXiv:0903.2527] [SPIRES].

[31] X.-H. Ge, Y. Matsuo, F.-W. Shu, S.-J. Sin and T. Tsukioka, Viscosity Bound, Causality Violation and Instability with Stringy Correction and Charge, JHEP 10 (2008) 009 [arXiv: 0808.2354] [SPIRES].

[32] M.F. Paulos, Transport coefficients, membrane couplings and universality at extremality, JHEP 02 (2010) 067 [arXiv: 0910.4602] [SPIRES].

[33] R.-G. Cai, Y. Liu and Y.-W. Sun, Transport Coefficients from Extremal Gauss-Bonnet Black Holes, JHEP 04 (2010) 090 [arXiv: 0910.4705] [SPIRES].

[34] C.-M. Chen and D.-W. Pang, Holography of Charged Dilaton Black Holes in General Dimensions, JHEP 06 (2010) 093 [arXiv: 1003.5064] [SPIRES].

[35] R.-G. Cai, Z.-Y. Nie and Y.-W. Sun, Shear Viscosity from the Effective Coupling of Gravitons, arXiv: 1006.0539 [SPIRES].

[36] S.S. Pal, $\eta / s$ at finite coupling, Phys. Rev. D 81 (2010) 045005 [arXiv:0910.0101] [SPIRES].

[37] I.P. Neupane, Black Holes, Entropy Bound and Causality Violation, Int. J. Mod. Phys. A 24 (2009) 3584 [arXiv:0904.4805] [SPIRES].

[38] M. Taylor, More on counterterms in the gravitational action and anomalies, hep-th/0002125 [SPIRES].

[39] K. Skenderis, Lecture notes on holographic renormalization, Class. Quant. Grav. 19 (2002) 5849 [hep-th/0209067] [SPIRES]. 
[40] J.H. Schwarz, Dilaton-axion symmetry, hep-th/9209125 [SPIRES].

[41] G.W. Gibbons, R. Kallosh and B. Kol, Moduli, scalar charges and the first law of black hole thermodynamics, Phys. Rev. Lett. 77 (1996) 4992 [hep-th/9607108] [SPIRES].

[42] D. Astefanesei, K. Goldstein and S. Mahapatra, Moduli and (un)attractor black hole thermodynamics, Gen. Rel. Grav. 40 (2008) 2069 [hep-th/0611140] [SPIRES].

[43] H. Nastase, Introduction to AdS-CFT, arXiv:0712.0689 [SPIRES].

[44] E. Witten, Anti-de Sitter space and holography, Adv. Theor. Math. Phys. 2 (1998) 253 [hep-th/9802150] [SPIRES].

[45] S.S. Gubser, I.R. Klebanov and A.M. Polyakov, Gauge theory correlators from non-critical string theory, Phys. Lett. B 428 (1998) 105 [hep-th/9802109] [SPIRES].

[46] D.T. Son and A.O. Starinets, Minkowski-space correlators in AdS/CFT correspondence: Recipe and applications, JHEP 09 (2002) 042 [hep-th/0205051] [SPIRES].

[47] C.P. Herzog and D.T. Son, Schwinger-Keldysh propagators from AdS/CFT correspondence, JHEP 03 (2003) 046 [hep-th/0212072] [SPIRES].

[48] D. Astefanesei, N. Banerjee and S. Dutta, (Un)attractor black holes in higher derivative AdS gravity, JHEP 11 (2008) 070 [arXiv:0806.1334] [SPIRES].

[49] H.K. Kunduri, J. Lucietti and H.S. Reall, Near-horizon symmetries of extremal black holes, Class. Quant. Grav. 24 (2007) 4169 [arXiv: 0705.4214] [SPIRES].

[50] D. Astefanesei and H. Yavartanoo, Stationary black holes and attractor mechanism, Nucl. Phys. B 794 (2008) 13 [arXiv: 0706.1847] [SPIRES].

[51] S. Ferrara, R. Kallosh and A. Strominger, $N=2$ extremal black holes, Phys. Rev. D 52 (1995) 5412 [hep-th/9508072] [SPIRES].

[52] A. Sen, Black Hole Entropy Function and the Attractor Mechanism in Higher Derivative Gravity, JHEP 09 (2005) 038 [hep-th/0506177] [SPIRES].

[53] A. Sen, Entropy function for heterotic black holes, JHEP 03 (2006) 008 [hep-th/0508042] [SPIRES].

[54] K. Goldstein, N. Iizuka, R.P. Jena and S.P. Trivedi, Non-supersymmetric attractors, Phys. Rev. D 72 (2005) 124021 [hep-th/0507096] [SPIRES].

[55] D. Astefanesei, K. Goldstein, R.P. Jena, A. Sen and S.P. Trivedi, Rotating attractors, JHEP 10 (2006) 058 [hep-th/0606244] [SPIRES].

[56] L. Andrianopoli, R. D'Auria, S. Ferrara and M. Trigiante, Extremal black holes in supergravity, Lect. Notes Phys. 737 (2008) 661 [hep-th/0611345] [SPIRES].

[57] A. Sen, Black Hole Entropy Function, Attractors and Precision Counting of Microstates, Gen. Rel. Grav. 40 (2008) 2249 [arXiv:0708.1270] [SPIRES].

[58] S. Bellucci, S. Ferrara, R. Kallosh and A. Marrani, Extremal Black Hole and Flux Vacua Attractors, Lect. Notes Phys. 755 (2008) 115 [arXiv:0711.4547] [SPIRES].

[59] S. Bellucci, S. Ferrara and A. Marrani, Attractors in Black, Fortsch. Phys. 56 (2008) 761 [arXiv: 0805.1310] [SPIRES].

[60] S. Ferrara, K. Hayakawa and A. Marrani, Lectures on Attractors and Black Holes, Fortsch. Phys. 56 (2008) 993 [arXiv:0805.2498] [SPIRES]. 
[61] D. Astefanesei, H. Nastase, H. Yavartanoo and S. Yun, Moduli flow and non-supersymmetric AdS attractors, JHEP 04 (2008) 074 [arXiv:0711.0036] [SPIRES].

[62] K. Goldstein, S. Kachru, S. Prakash and S.P. Trivedi, Holography of Charged Dilaton Black Holes, JHEP 08 (2010) 078 [arXiv:0911.3586] [SPIRES].

[63] R.H. Price and K.S. Thorne, Membrane viewpoint on black holes: Properties and evolution of the stretched horizon, Phys. Rev. D 33 (1986) 915 [SPIRES].

[64] M. Parikh and F. Wilczek, An action for black hole membranes, Phys. Rev. D 58 (1998) 064011 [gr-qc/9712077] [SPIRES].

[65] J.M. Maldacena, Eternal black holes in Anti-de-Sitter, JHEP 04 (2003) 021 [hep-th/0106112] [SPIRES].

[66] K. Skenderis and B.C. van Rees, Real-time gauge/gravity duality, Phys. Rev. Lett. 101 (2008) 081601 [arXiv:0805.0150] [SPIRES].

[67] K. Skenderis and B.C. van Rees, Real-time gauge/gravity duality: Prescription, Renormalization and Examples, JHEP 05 (2009) 085 [arXiv:0812.2909] [SPIRES].

[68] P. Kovtun, D.T. Son and A.O. Starinets, Holography and hydrodynamics: Diffusion on stretched horizons, JHEP 10 (2003) 064 [hep-th/0309213] [SPIRES].

[69] N. Banerjee and S. Dutta, Near-Horizon Analysis of $\eta / s$, Nucl. Phys. B 845 (2011) 165 [arXiv:0911.0557] [SPIRES].

[70] E.I. Buchbinder and A. Buchel, The Fate of the Sound and Diffusion in Holographic Magnetic Field, Phys. Rev. D 79 (2009) 046006 [arXiv:0811.4325] [SPIRES].

[71] R. Brustein and A.J.M. Medved, The ratio of shear viscosity to entropy density in generalized theories of gravity, Phys. Rev. D 79 (2009) 021901 [arXiv:0808.3498] [SPIRES].

[72] K. Hanaki, K. Ohashi and Y. Tachikawa, Supersymmetric completion of an $R^{2}$ term in five-dimensional supergravity, Prog. Theor. Phys. 117 (2007) 533 [hep-th/0611329] [SPIRES].

[73] S. Cremonini, K. Hanaki, J.T. Liu and P. Szepietowski, Higher derivative effects on $\eta / s$ at finite chemical potential, Phys. Rev. D 80 (2009) 025002 [arXiv: 0903.3244] [SPIRES].

[74] R.M. Wald, Black hole entropy is the Noether charge, Phys. Rev. D 48 (1993) 3427 [gr-qc/9307038] [SPIRES].

[75] S. Dutta and R. Gopakumar, On Euclidean and Noetherian entropies in AdS space, Phys. Rev. D 74 (2006) 044007 [hep-th/0604070] [SPIRES].

[76] E. D'Hoker and P. Kraus, Magnetic Field Induced Quantum Criticality via new Asymptotically AdS $S_{5}$ Solutions, Class. Quant. Grav. 27 (2010) 215022 [arXiv:1006.2573] [SPIRES].

[77] S. Nakamura, H. Ooguri and C.-S. Park, Gravity Dual of Spatially Modulated Phase, Phys. Rev. D 81 (2010) 044018 [arXiv:0911.0679] [SPIRES].

[78] H. Ooguri and C.-S. Park, Holographic End-Point of Spatially Modulated Phase Transition, Phys. Rev. D 82 (2010) 126001 [arXiv:1007.3737] [SPIRES].

[79] D. Astefanesei, N. Banerjee and S. Dutta, work in progress.

[80] D. Astefanesei and Y.K. Srivastava, CFT Duals for Attractor Horizons, Nucl. Phys. B 822 (2009) 283 [arXiv:0902.4033] [SPIRES]. 
[81] N. Banerjee and S. Dutta, Nonlinear Hydrodynamics from Flow of Retarded Green's Function, JHEP 08 (2010) 041 [arXiv: 1005.2367] [SPIRES].

[82] E.I. Buchbinder and A. Buchel, Relativistic Conformal Magneto-Hydrodynamics from Holography, Phys. Lett. B 678 (2009) 135 [arXiv:0902.3170] [SPIRES].

[83] J.-Y. Ollitrault, Relativistic hydrodynamics, Eur. J. Phys. 29 (2008) 275 [arXiv:0708.2433] [SPIRES].

[84] M. Mia, K. Dasgupta, C. Gale and S. Jeon, Five Easy Pieces: The Dynamics of Quarks in Strongly Coupled Plasmas, Nucl. Phys. B 839 (2010) 187 [arXiv:0902.1540] [SPIRES]. 